\title{
Fuzzy Mathematical Models of Type-1 and Type-2 for Computing the Parameters and Its Applications
}

\author{
Rana Waleed Hndoosh \\ Dept. of Software Engineering, \\ College of Computers Sciences and \\ Mathematics, Mosul University, Iraq.
}

\author{
M. S. Saroa \\ Dept. of Mathematics, Maharishi \\ Markandeshawar University, \\ Mullana, 133207, India
}

\author{
Sanjeev Kumar \\ Dept. of Mathematics, Dr. B. R. \\ Ambedkar University, Khandari \\ Campus, Agra-282002, India.
}

\begin{abstract}
This work provides mathematical formulas and algorithm in order to calculate the derivatives that being necessary to perform Steepest Descent models to make T1 and T2 FLSs much more accessible to FLS modelers. It provides derivative computations that are applied on different kind of MFs, and some computations which are then clarified for specific MFs. We have learned how to model T1 FLSs when a set of training data is available and provided an application to derive the Steepest Descent models that depend on trigonometric function (SDTFM). This work, also focused on an interval type-2 non-singleton type-2 FLS (IT2 NS-T2 FLS) in order to determine how to assign all the parameters of the antecedent and consequent MFs using the set of $n$ input-output and build mathematical formulas to calculate the derivatives $\partial \cosh (\alpha) / \partial \theta$ depend on general formula of SDTFM. Additionally, we showed how to complete the calculations for input measurement and antecedent Gaussian primary MFs with uncertain standard deviations and means.
\end{abstract}

\section{General Terms}

Fuzzy modeling, fuzzy logic system, uncertainty

\section{Keywords}

Type-2 fuzzy sets, interval type-2 membership functions, type-2 fuzzy logic system, steepest descent models, interval type-2 non-singleton type-2 FLS, derivative, uncertainty.

\section{INTRODUCTION}

In many mathematical and engineering problems, the computation of certain solutions depends on the availability of exact values for the variables of model equations. Because the existing information usually is incomplete, inaccurate, fuzzy or linguistic, then the accurate values cannot be obtained. Therefore, it is necessary to introduce uncertain variables for modeling the available information [1], [22]. Both T1 FLS and T2 FLS include fuzzifier, rule-base, fuzzy inference engine, and output processor [2]. The output processor in T2 FLS includes type-reducer and defuzzifier while the output processor in T1 FLS includes a crisp number from the defuzzifier, [28], [4-8]. If-then rules, but its antecedent describes a T2 or consequent sets are now T2, [13], [18], [25]. T2 FLSs can be used when the cases are so uncertain to determine exact membership degrees such as when training data is corrupted by noise [3], [16], [17]. The most popular one to date, uses back propagation models (steepest descent models) for tuning all model parameters, which require the computation of first derivatives of an objective function with respect to each model parameter for making T2 FLSs much more accessible to FLS designers [23]. When all T2 FSs are modeled as interval sets, and then we obtain an IT2 FLS. We have focused on an interval type-2 non-singleton type-2 FLS (IT2 NS-T2 FLSs) [9], [26]. The T1 FLS is described using a fuzzy basis function extension that is useful for computing the output of that FLS, and used during its model for computing derivatives of an objective function with respect to MF parameters, [19]. By "model", we specify the parameters that describe the interval T2 FLS [10], [11]. A T2 FLS model method builds how to determine all the parameters of the antecedent and consequent MFs using the training pairs [30], [4-8].

In the first part of work, we will focus on rule-based FLSs when no uncertainties are present [21], [27]. This is similar to first studying deterministic systems before studying random systems [12]. Then we will learn about extension of rulebased FLSs, ones that can directly model uncertainties [29]. The major purpose of this work is to learn how to model nonsingleton type-1 fuzzy logic systems (NS-T1 FLSs) when a set of training data is available. Recount how many model parameters there can be in a specific model and describe the relation of that number to the number of possible rules in the NS-T1 FLS [20]. Explain how to calculate the derivatives that are needed for the backpropagation, such as Steepest Descent model that depend on trigonometric function (SDTFM), for updating the MF parameters. The training data is used to tune the input measurement, antecedent, and consequent MF parameters. Here mathematical formulas are built to calculate the derivatives $\partial \cosh (j) / \partial \theta$.

The structure of this work provides an introduction in this Section. In Section 2, we will learn how to model T1 FLSs when a set of training data is available [3], [2]; Section 3 provides an application to derive the SDTFM, [19], [15]. Generalized bell-shaped MF is chosen for the antecedent and the consequent [24]. Section 4, focused on an IT2 NS-T2 FLS in order to determine how to assign all the parameters of the antecedent and consequent MFs using the set of $n$ inputoutput and build mathematical formulas to calculate the derivatives $\partial \cosh (\alpha) / \partial \theta$ depend on general formula of SDTFM [13]. Also, provides general formulas for the left and right end-points of the type reduced set [26]. Section 5 provides computation of $\partial \cosh (\alpha) / \partial \theta_{i, k}^{l}$ for antecedent and consequent parameters for derivatives of $\partial \cosh (\alpha)$ with respect to antecedent MF parameters and provided an algorithm of the derivatives for antecedent parameters to calculate $\partial \cosh (\alpha) / \partial \theta_{i, k}^{l}$ [19], [13]. Section 6 provides an application in order to calculate $\partial \underline{\mu}_{\hat{O}_{i}^{l}}\left(\underline{x}_{i, s}^{l}\right) / \partial \theta_{i, k}^{l}$, and $\partial \bar{\mu}_{\hat{o}_{i}^{l}}\left(\bar{x}_{i, s}^{l}\right) / \partial \theta_{i, k}^{l}$ for antecedent Gaussian primary MFs with uncertain means, and input measurement Gaussian primary 
MFs with uncertain standard deviations [30]. Finally, Section 7 , conclusions are presented, and handles the derivatives of the left end-point of the type-reduced set.

\section{MODEL TYPE-1 FUZZY LOGIC SYSTEMS}

The main purpose of this Section is to learn how to model T1 FLSs when a set of training data is available. By "model", we mean specify the parameters that describe the FLS. We collect all of the equations that are needed to implement NS-T1 FLSs, [16], [17]. These equations require the modeler to make many choices. The general equations for inference system as follows [29], [19]:

$\mu_{O_{i}^{l}}\left(x_{i, s}^{l}\right)=\sup _{x_{i} \in X_{i}} \mu_{X_{i}}\left(x_{i}\right) \star \mu_{A_{i}^{l}}\left(x_{i}\right)$,

where,

$x_{i, s}^{l}=\arg \left[\sup _{x_{i} \in X_{i}} \mu_{X_{i}}\left(x_{i}\right) \star \mu_{A_{i}^{l}}\left(x_{i}\right)\right]$

$\mu_{D^{l}}(y)=\mu_{B^{l}}(y) \star T_{i=1}^{n} \mu_{O_{i}^{l}}\left(x_{i, s}^{l}\right)$

The input-output equation, $y(\mathrm{x})$, for the FLS is given by:

$f_{n s}(\mathrm{x})=\frac{\sum_{l=1}^{M} \bar{y}^{l} \min _{i=1, \ldots, n} \mu_{A_{i}^{l}}\left(x_{i}\right)}{\sum_{l=1}^{M} \min _{i=1, \ldots, n} \mu_{A_{i}^{l}}\left(x_{i}\right)}=\sum_{l=1}^{M} \bar{y}^{l} \varphi_{l}(\mathrm{x})$

There are many ways to optimize a function [18], but we will describe a very popular way that uses the value of the function being optimized and its first derivative. Methods that use this information are called steepest descent (SDM). Here suppose that the function being minimized depends on the parameter $\theta$, and the function is denoted $J(\theta)$ that is called an objective function [1], [22]. We have a mathematical formula for $J(\theta)$, and we do not know the shape of $J(\theta)$, but available only a set of training data $\left\{\left(x^{(j)}, y^{(j)}\right), j=1, \ldots, n\right\}$, here $J=J(D, \theta)$. Let $D_{T}$ is used by the $S D M$ because that model is based on minimizing $D_{T}=J\left(D_{T}, \theta\right)$. The general structure of a $S D M$ for minimizing objective function is as [19], [20]:

$\theta_{j+1}=\theta_{j}-\gamma_{\theta}\left(\right.$ derivatives of $\left.\left.J\left(D_{T}, \theta\right)\right|_{\theta_{j}}\right)$,

where $\gamma_{\theta}$ is a learning parameter, and $j=0,1, \ldots$. In this tuning procedure, a trigonometric function is used, as:

$J\left(D_{T}, \theta\right)=\cosh \left(D_{T}, \theta_{j}\right)$,

where

$$
\begin{aligned}
& \cosh \left(D_{T}, \theta\right)= \\
& \frac{\left(\left(\frac{\left[y\left(D_{T}, \theta\right)-y^{(j)}\left(D_{T}\right)\right]^{2}}{2}\right)^{-1}+\left(\frac{\left[y\left(D_{T}, \theta\right)-y^{(j)}\left(D_{T}\right)\right]^{2}}{2}\right)\right)}{2} \\
& =\frac{1}{\left[y\left(D_{T}, \theta\right)-y^{(j)}\left(D_{T}\right)\right]^{2}}+\frac{\left[y\left(D_{T}, \theta\right)-y^{(j)}\left(D_{T}\right)\right]^{2}}{4} \\
& y\left(D_{T}, \theta\right)=f\left(D_{T}, \theta\right)
\end{aligned}
$$

From (8), note that $f\left(D_{T}, \theta\right)$ is the output of a T1 FLS. It is easy to calculate the derivatives of $J\left(D_{T}, \theta\right)$ that are needed in (5), using (6)-(8),

$$
\begin{aligned}
& \frac{\partial J\left(D_{T}, \theta\right)}{\partial \theta}=\frac{\partial \cosh \left(D_{T}, \theta\right)}{\partial \theta} \\
& \quad=\left(\frac{1}{2}\left[y\left(D_{T}, \theta\right)-y^{(j)}\left(D_{T}\right)\right]-\frac{2}{\left[y\left(D_{T}, \theta\right)-y^{(j)}\left(D_{T}\right)\right]^{3}}\right) \frac{\partial y\left(D_{T}, \theta\right)}{\partial \theta} \\
& =\left(\begin{array}{c}
\frac{1}{2}\left[y\left(D_{T}, \theta\right)-y^{(j)}\left(D_{T}\right)\right]- \\
\frac{2}{\left[y\left(D_{T}, \theta\right)-y^{(j)}\left(D_{T}\right)\right]^{3}}
\end{array}\right) \frac{\partial f\left(D_{T}, \theta\right)}{\partial \theta}
\end{aligned}
$$

For follow up, the specified FLS choices mentioned above must be made. Those choices will let us assign analytical formulas for $\partial f\left(D_{T}, \theta\right) / \partial \theta$. We continue to complete these computations for a specific set of choices through an application in the next Section [24], [18].

\section{APPLICATION}

This Section will derive the Steepest Descent model that depends on trigonometric function (SDTFM). Note that the following generalized bell-shaped MF is chosen for the antecedent and the consequent [30],

$\mu(x)=\frac{1}{1+\left(\frac{x-m}{\sigma}\right)^{2 s}}$,

in which $m, \sigma$ are used to adjust to vary the center and width of the membership function, and $s$ denotes the slop at the cross points. The final implementation of input-output equation for the FLS requires choices to be made about the MFs, where generalized bell-shaped antecedent and input MFs respectively are given as the following:

$$
\begin{aligned}
& \mu_{A_{i}^{l}}\left(x_{i}\right)=\frac{1}{1+\left(\frac{x_{i}-m_{A_{i}^{l}}}{\sigma_{A_{i}^{l}}}\right)^{2 s_{A_{i}^{l}}}}, \quad l=1, \ldots, M \\
& \mu_{X_{i}}\left(x_{i}\right)=\frac{1}{1+\left(\frac{x_{i}-m_{X_{i}}}{\sigma_{X_{i}}}\right)^{2 s_{X_{i}}}}, \quad i=1, \ldots, n ., \\
& \mu_{O_{i}^{l}}\left(x_{i, S}^{l}\right)=\frac{1}{1+\left(\frac{m_{X_{i}}-m_{A_{i}^{l}}}{\sigma_{X_{i}}+\sigma_{A_{i}^{l}}}\right)^{2\left(s_{X_{i}}+s_{A_{i}^{l}}\right)} .}
\end{aligned}
$$

Equations (4) and (13) perform a non-singleton T1 FLS, the parameter $\theta=\hat{y}^{l}, m_{X_{i}}, m_{A_{i}^{l}}, \sigma_{X_{i}}, \sigma_{A_{i}^{l}}, s_{X_{i}}$ or $s_{A_{i}^{l}}$.

The certain parts of the computations of $\partial J(\theta) / \partial \theta$, where $J(\theta)=\cosh (j)=\frac{1}{\left[f_{n s}\left(x^{(j)}\right)-y^{(j)}\right]^{2}}+\frac{\left[f_{n s}\left(x^{(j)}\right)-y^{(j)}\right]^{2}}{4}$, are as the following:

$$
\begin{aligned}
\frac{\partial J(\theta)}{\partial \theta} & =\frac{\partial}{\partial \theta}\left\{\frac{1}{\left[f_{n s}\left(x^{(j)}\right)-y^{(j)}\right]^{2}}+\frac{\left[f_{n s}\left(x^{(j)}\right)-y^{(j)}\right]^{2}}{4}\right\} \\
& =\left(\begin{array}{l}
\frac{1}{2}\left[f_{n s}\left(x^{(j)}\right)-y^{(j)}\right] \\
-\frac{2}{\left[f_{n s}\left(x^{(j)}\right)-y^{(j)}\right]^{3}}
\end{array}\right) \frac{\partial}{\partial \theta} f_{n s}\left(\mathrm{x}^{(j)}\right)
\end{aligned}
$$


where,

$$
\begin{aligned}
& f_{n s}\left(x^{(j)}\right)=\sum_{l=1}^{M} \hat{y}^{l} \varphi_{l}\left(x^{(j)}\right)=\sum_{l=1}^{M} \hat{y}^{l} \frac{\min _{i=1, \ldots, n} \mu_{O_{i}^{l}}\left(x_{i, s}^{l}\right)}{\sum_{l=1}^{M} \min _{i=1, \ldots, n} \mu_{O_{i}^{l}}\left(x_{i, s}^{l}\right)}
\end{aligned}
$$

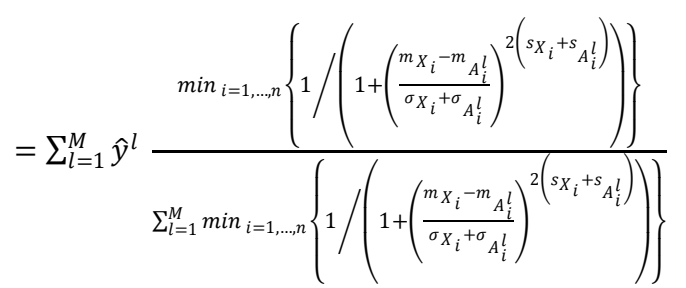

Derivative of the output of non-singleton T1 FLS that given by (15) with respect to each one of the parameter $\theta$ as the follows:

1. When $\theta=\hat{y}^{l}$

$\frac{\partial}{\partial \hat{y}^{l}} f_{n s}\left(x^{(j)}\right)=\varphi_{l}\left(\mathrm{x}^{(j)}\right)$,

so that

$$
\begin{aligned}
\hat{y}_{j+1}^{l} & =\hat{y}_{j}^{l}-\gamma_{\hat{y}^{l}}\left(\frac{\partial}{\partial \hat{y}^{l}} J\left(\hat{y}^{l}\right)\right) \\
& =\hat{y}_{j}^{l}-\gamma_{\hat{y}^{l}}\left(\left(\begin{array}{c}
\frac{1}{2}\left[f_{n s}\left(\mathrm{x}^{(j)}\right)-y^{(j)}\right] \\
-\frac{2}{\left[f_{n s}\left(\mathrm{x}^{(j)}\right)-y^{(j)}\right]^{3}}
\end{array}\right) \varphi_{l}\left(\mathrm{x}^{(j)}\right)\right)
\end{aligned}
$$

2. When $\theta=m_{X_{i}}$,

$$
\begin{aligned}
& f_{n s}\left(x^{(j)}\right)=\frac{\sum_{l=1}^{M} \hat{y}^{l} w^{l}}{\sum_{l=1}^{M} w^{l}}
\end{aligned}
$$

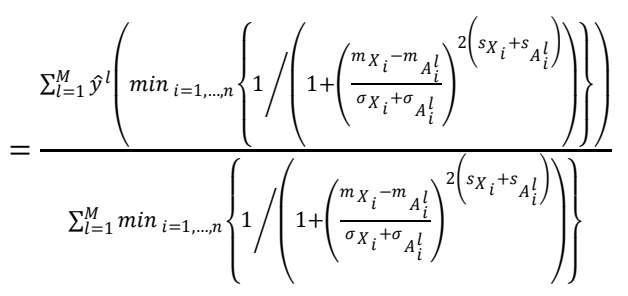

Therefore,

$$
\begin{aligned}
\frac{\partial f_{n s}}{\partial m_{X_{i}}} & =\frac{\partial f_{n s}}{\partial w^{l}} \cdot \frac{\partial w^{l}}{\partial m_{X_{i}}} \\
\frac{\partial f_{n s}}{\partial w^{l}} & =\frac{\left(\sum_{l=1}^{M} w^{l}\right) \cdot \frac{\partial\left(\sum_{l=1}^{M} \hat{y}^{l} w^{l}\right)}{\partial w^{l}}-\left(\sum_{l=1}^{M} \hat{y}^{l} w^{l}\right) \cdot \frac{\partial\left(\sum_{l=1}^{M} w^{l}\right)}{\partial w^{l}}}{\left(\sum_{l=1}^{M} w^{l}\right)^{2}} \\
& =\frac{\hat{y}^{l}\left(\sum_{l=1}^{M} w^{l}\right)-\left(\sum_{l=1}^{M} \hat{y}^{l} w^{l}\right)}{\left(\sum_{l=1}^{M} w^{l}\right)^{2}}=\frac{\hat{y}^{l}-f_{n s}\left(x^{(j)}\right)}{\sum_{l=1}^{M} w^{l}}
\end{aligned}
$$

And

$$
\frac{\partial w^{l}}{\partial m_{X_{i}}}=\frac{\partial}{\partial m_{X_{i}}}\left(\min _{i=1, \ldots, n}\left\{1 /\left(1+\left(\frac{m_{X_{i}}-m_{A_{i}^{l}}}{\sigma_{X_{i}}+\sigma_{A_{i}^{l}}}\right)^{2\left(s_{X_{i}}+s_{A_{i}^{l}}\right)}\right)\right\}\right)
$$

$$
\begin{aligned}
& =\min _{i=1, \ldots, n}\left\{1 /\left(1+\left(\frac{m_{X_{i}}-m_{A_{i}^{l}}}{\sigma_{X_{i}}+\sigma_{A_{i}^{l}}}\right)^{2\left(s_{X_{i}}+s_{A_{i}^{l}}\right)}\right)\right\} *
\end{aligned}
$$

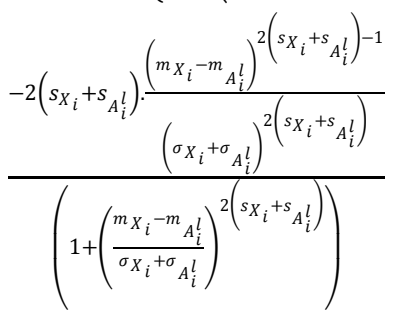

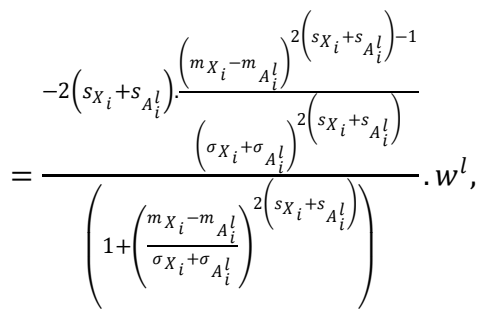

thus,

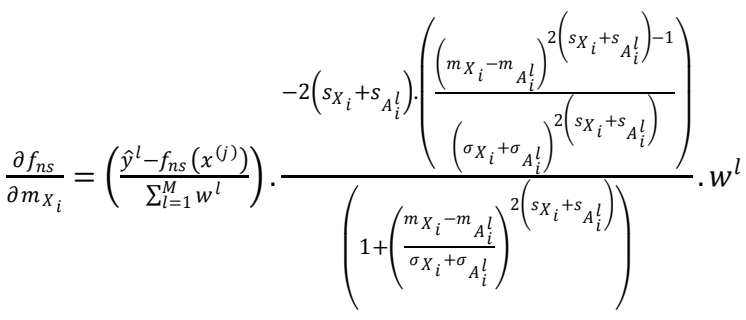

Consequently,

$$
\begin{aligned}
& \left(m_{X_{i}}\right)_{j+1}=\left(m_{X_{i}}\right)_{j}-\gamma_{m}\left(\begin{array}{c}
\frac{1}{2}\left[f_{n s}\left(\mathrm{x}^{(j)}\right)-y^{(j)}\right] \\
-\frac{2}{\left[f_{n s}\left(\mathrm{x}^{(j)}\right)-y^{(j)}\right]^{3}}
\end{array}\right) \cdot \frac{\partial f_{n s}}{\partial m_{X_{i}}} \\
& \left(m_{X_{i}}\right)_{j+1}=\left(m_{X_{i}}\right)_{j}-\gamma_{m} *
\end{aligned}
$$

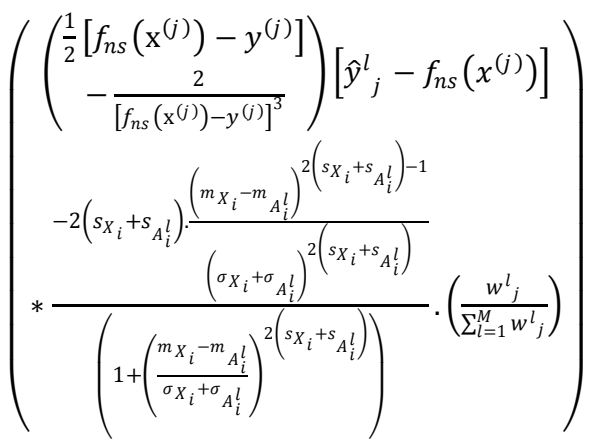

From (15) and (18), we note that,

$\frac{w_{j}^{l}}{\sum_{l=1}^{M} w_{j}^{l}}=\varphi_{l}\left(x^{(j)}\right)$.

Replacing equation (23) into the one just before it, then we reach the SDTFM for updating $m_{j}^{l}$ as the following:

$$
\left(m_{X_{i}}\right)_{j+1}=\left(m_{X_{i}}\right)_{j}-\gamma_{m} *
$$


International Journal of Computer Applications (0975 - 8887)

Volume 104 - No.14, October 2014

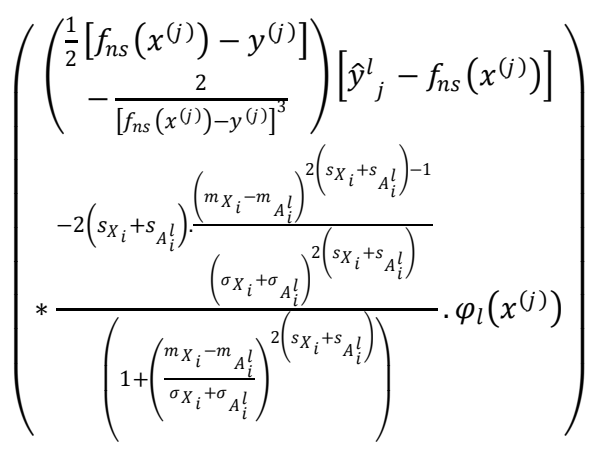

(24)

Similarly, when $\theta=m_{A_{i}^{l}}$, we obtain,

$\left(m_{A_{i}^{l}}\right)_{j+1}=\left(m_{A_{i}^{l}}\right)_{j}-\gamma_{m} *$

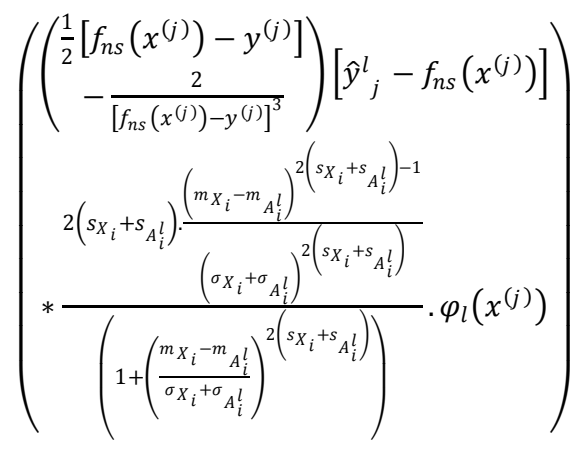

3. When $\theta=\sigma_{X_{i}}$,

The derivation of $\partial f_{n S} / \partial \sigma_{X_{i}}$ is just like the derivation of $\partial f_{n s} / \partial m_{X_{i}}$, therefore, we calculate,

$\frac{\partial f_{n s}}{\partial \sigma_{X_{i}}}=\frac{\partial f_{n s}}{\partial w^{l}} \cdot \frac{\partial w^{l}}{\partial \sigma_{X_{i}}}$,

(26)

where $\partial f_{n s} / \partial w^{l}$ has been computed through (19). So, we need only the new computation of $\partial w^{l} / \partial \sigma_{X_{i}}$.

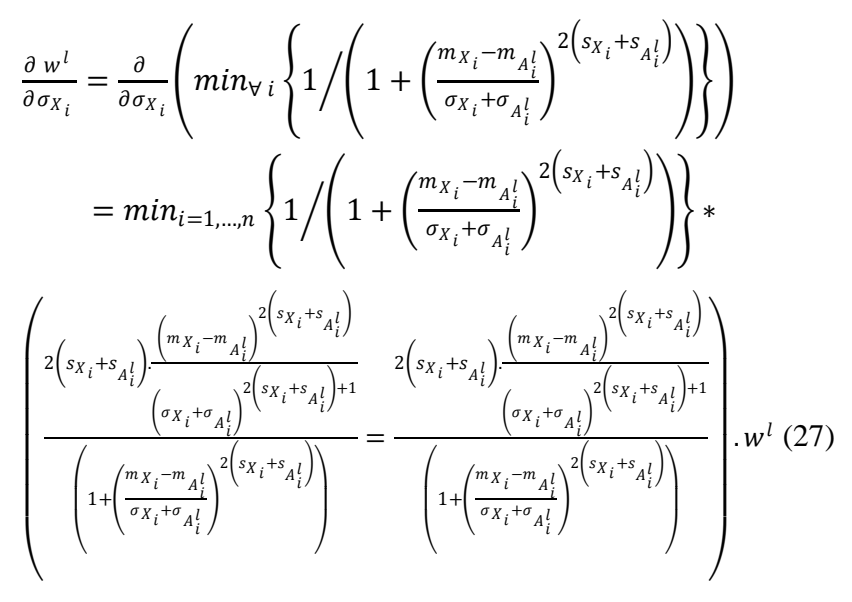

Thus,

$\frac{\partial f_{n s}}{\partial \sigma_{j}^{l}}=\left(\frac{\hat{y}^{l}-f_{n s}\left(x^{(j)}\right)}{\sum_{l=1}^{M} w^{l}}\right) *$

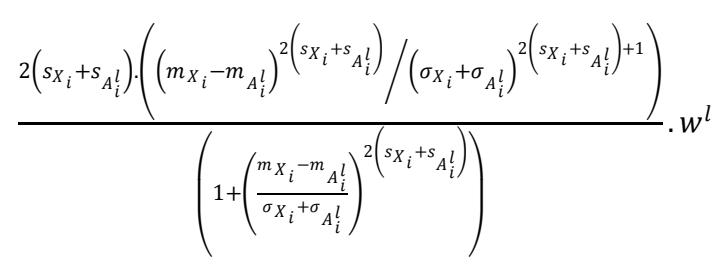

Therefore,

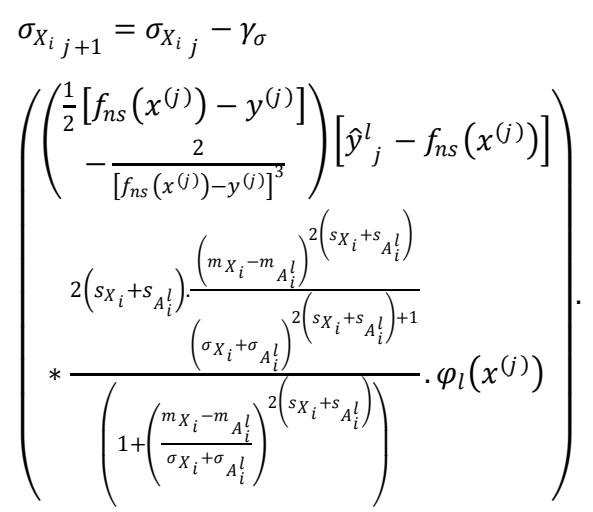

Similarly, when $\theta=\sigma_{A_{i}^{l}}$, we obtain,

$\sigma_{A_{i j+1}^{l}}=\sigma_{A_{i j}^{l}}-\gamma_{\sigma} *$

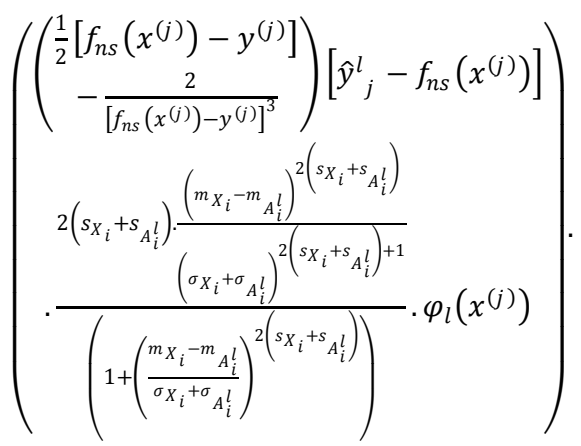

4. When $\theta=s_{X_{i}}$,

The derivation of $\partial f_{n s} / \partial s_{X_{i}}$ is just like the derivation of $\partial f_{n s} / \partial \sigma_{X_{i}}$, and we then calculate

$\frac{\partial f_{n s}}{\partial s_{X_{i}}}=\frac{\partial f_{n s}}{\partial w^{l}} \cdot \frac{\partial w^{l}}{\partial s_{X_{i}}}$,

where $\partial f_{n s} / \partial w^{l}$ has been computed through (19). We only need the new computation of $\partial w^{l} / \partial s_{X_{i}}$.

$$
\begin{aligned}
& \frac{\partial w^{l}}{\partial s_{X_{i}}}=\frac{\partial}{\partial s_{X_{i}}}\left(\min _{i=1, \ldots, n}\left\{1 /\left(1+\left(\frac{m_{X_{i}}-m_{A_{i}^{l}}}{\sigma_{X_{i}}+\sigma_{A_{i}^{l}}}\right)^{2\left(s_{X_{i}}+s_{A_{i}^{l}}\right)}\right)\right\}\right) \\
& =\min _{i=1, \ldots, n}\left\{1 /\left(1+\left(\frac{m_{X_{i}}-m_{A_{i}^{l}}}{\sigma_{X_{i}}+\sigma_{A_{i}^{l}}}\right)^{2\left(s_{X_{i}}+s_{A_{i}^{l}}\right)}\right)\right\} *
\end{aligned}
$$

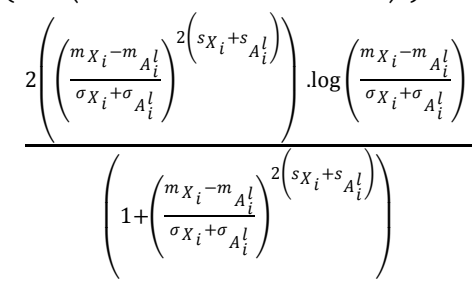

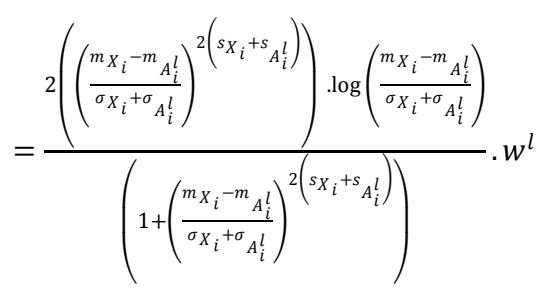

Thus,

20 


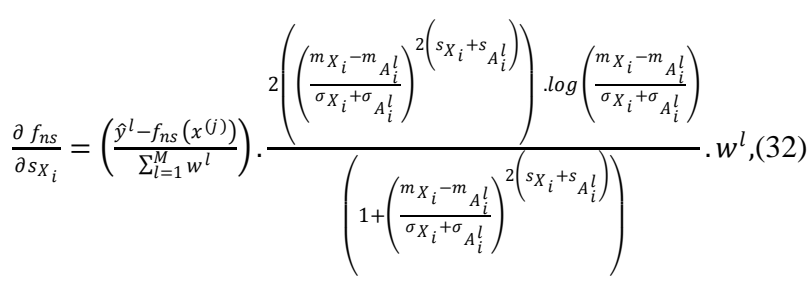

Therefore,

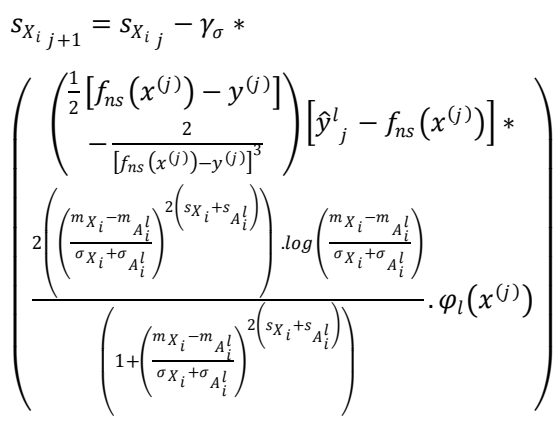

Similarly, when $\theta=s_{A_{i}^{l}}$, we obtain,

$\left(s_{A_{i}^{l}}\right)_{j+1}=s_{A_{i j}^{l}}-\gamma_{\sigma} *$

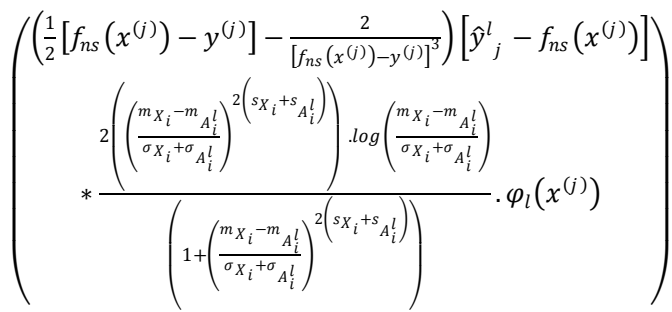

\section{MODEL INTERVAL TYPE-2 NON- SINGLETON TYPE-2 FUZZY LOGIC SYSTEM}

This Section will model an interval type-2 non-singleton type2 fuzzy logic system (IT2 NS-T2 FLS). We are given a set of data training pairs, $\left\{\left(x^{(1)}, y^{(1)}\right),\left(x^{(2)}, y^{(2)}\right), \ldots,\left(x^{(n)}, y^{(n)}\right)\right\}$, where $x$ is an input vector and $y$ is the scalar output of an IT2 FLS. There are many types of IT2 FLS; but we focused on an IT2 NS-T2 FLS [29]. The rule antecedents and consequent of FLS described by IT2 FSs and the inputs that activate the FLS are IT2 FSs [10], [11]. The MFs is denoted by $\mu_{X_{i}}\left(x_{i}\right)$ for all input $x_{i}$, with lower MFs $\underline{\mu}_{X_{i}}\left(x_{i}\right)$ and upper MFs $\bar{\mu}_{X_{i}}\left(x_{i}\right)$, and the aim is to specify the type-2 FLS by using the training data. This model determines how to assign all the parameters of the antecedent and consequent MFs using the set of $n$ input-output. Suppose a general structure that tuned all MF parameters given by the formula:

$\theta_{j+1}=\theta_{j}-\left.\gamma_{\theta} \frac{\partial \cosh (\alpha)}{\partial \theta}\right|_{j}$,

where $\theta$ is a model for any parameter of the FLS, $\left.\gamma_{\theta} \frac{\partial \cosh (\alpha)}{\partial \theta}\right|_{n}$ denotes that after taking the derivative with respect to a specified. We have to replace all remaining $\theta$ values by $\theta_{j}$, and $\cosh (\alpha)=\left(\mathrm{e}^{-(\alpha)}+\mathrm{e}^{(\alpha)}\right) / 2=$ $\left(\left(\mathrm{e}^{(\alpha)}\right)^{-1}+\mathrm{e}^{(\alpha)}\right) / 2$, in which $e^{(\alpha)}=\left[f_{n s}\left(x^{(\alpha)}\right)-y^{(\alpha)}\right]^{2} / 2, \quad \alpha=1, \ldots, n$,

Then,

$\cosh (\alpha)=$

$$
\begin{gathered}
\left(\left(\left[f_{n s}\left(x^{(\alpha)}\right)-y^{(\alpha)}\right]^{2} / 2\right)^{-1}+\left(\left[f_{n s}\left(x^{(\alpha)}\right)-y^{(\alpha)}\right]^{2} / 2\right)\right) / 2 \\
=\frac{1}{\left[f_{n s}\left(x^{(\alpha)}\right)-y^{(\alpha)}\right]^{2}}+\frac{\left[f_{n s}\left(x^{(\alpha)}\right)-y^{(\alpha)}\right]^{2}}{4}
\end{gathered}
$$

The aim of this part is to build mathematical formulas for calculate the derivatives $\partial \cosh (\alpha) / \partial \theta$. The kinds of primary MFs can be described mathematically such as Gaussians, triangles, trapezoids, etc [30]. We have chosen height typereduction because there is a specific appearance of antecedent and consequent MF parameters for it. Since the centroid of an IT2 FS is an IT1 FS [9], [12], and as sets are completely described by their left-end point $y_{l}$ and right-end point $y_{l}$; then, calculating the centroid of an IT2 FS just requires calculating those two end-points [2]. All the different types of type-reduction, $Y_{T-R}(x)$, can be expressed as, [13], [15], [17]:

$\left[y_{l}, y_{r}\right]=$

$$
=\int_{y^{1} \in\left[y_{l}^{1}, y_{r}^{1}\right]} \ldots \int_{y^{M} \in\left[y_{l}^{M}, y_{r}^{M}\right]} \int_{a^{1} \in\left[\underline{a}^{1}, \bar{a}^{1}\right]} \ldots \int_{a^{M} \in\left[\underline{a}^{M}, \bar{a}^{M}\right]}^{.}\left(1 / \frac{\int_{i=1}^{M} y^{i} a^{i}}{\int_{i=1}^{M} a^{i}}\right)
$$

Here focused on the hight type-reduction; hence, $y_{l}^{i}=y_{l}^{i}=$ $y^{i}$ be a single point in the consequent domain of the $i^{\text {th }}$ rule and treated as a consequent parameter, $\underline{a}^{i}$ and $\bar{a}^{i}$ are lower and upper firing degrees of the $i^{\text {th }}$ rule that contains antecedent MF parameters, and $M$ is a rules number. We always compute $y_{l}$ and $y_{r}$ using the Karnik-Mendel iterative procedures, [13], [14]. Therefore, reorder the $a^{i}$ accordingly and call them $b^{i}$ and we can be represented $y_{l}$ and $y_{r}$ as [28]:

$y_{l}=\frac{\sum_{i=1}^{M} y_{l}^{i} b_{l}^{i}}{\sum_{i=1}^{M} b_{l}^{i}}=\frac{\sum_{i=1}^{L} y_{l}^{i} \bar{b}^{i}+\sum_{i=L+1}^{M} y_{l}^{i} \underline{b}^{i}}{\sum_{i=1}^{L} \bar{b}^{i}+\sum_{i=L+1}^{M} \underline{b}^{i}}$,

And

$y_{r}=\frac{\sum_{i=1}^{M} y_{r}^{i} b_{r}^{i}}{\sum_{i=1}^{M} b_{r}^{i}}=\frac{\sum_{i=1}^{R} y_{r}^{i} \bar{b}^{i}+\sum_{i=R+1}^{M} y_{r}^{i} \underline{b}^{i}}{\sum_{i=1}^{R} \bar{b}^{i}+\sum_{i=R+1}^{M} \underline{b}^{i}}$.

Since $b_{l}^{i}, y_{l}^{i}, b_{r}^{i}$ and $y_{r}^{i}$ have been unordered during step-1 of the two iterative procedures of type-reduction, therefore, these formulas cannot be used as it is. We need to know exactly where specified antecedent and consequent MF parameters are located for computing the derivatives of $y_{l}$ and $y_{r}$ with respect to parameters of MF. It is very difficult to satisfy when $y_{l}$ and $y_{r}$ are not in rule-ordered format, therefore, we must first re-express (37) and (38) in rule-ordered format [11].

Now, let $A^{l}\left(\mathrm{x}^{\prime}\right)$ are denoted rule-ordered firing intervals, and we have described the rule-unordered firing intervals by $B^{l}\left(\mathrm{x}^{\prime}\right)$, as the following [20]:

$A^{l}\left(x^{\prime}\right)=\left[\underline{a}^{l}\left(x^{\prime}\right), \bar{a}^{l}\left(x^{\prime}\right)\right]=\left[\underline{a}^{l}, \bar{a}^{l}\right]$,

$B^{l}\left(x^{\prime}\right)=\left[\underline{b}^{l}, \bar{b}^{l}\right]$. 
Let $y_{l}$ and $y_{r}$ denote rule-unordered values as the following [3]:

$y_{l}=\left(y_{l}^{1}, \ldots, y_{l}^{M}\right)^{T}$ and $y_{r}=\left(y_{r}^{1}, \ldots, y_{r}^{M}\right)^{T}$,

$y_{l}=\frac{\sum_{i=1}^{L} y_{l}^{i} \bar{b}^{i}+\sum_{i=L+1}^{M} y_{l}^{i} \underline{b}^{i}}{\sum_{i=1}^{L} \bar{b}^{i}+\sum_{i=L+1}^{M} \underline{b}^{i}}, y_{r}=\frac{\sum_{i=1}^{R} y_{r}^{i} \bar{b}^{i}+\sum_{i=R+1}^{M} y_{r}^{i} \underline{b}^{i}}{\sum_{i=1}^{R} \bar{b}^{i}+\sum_{i=R+1}^{M} \underline{b}^{i}}$,

and $z_{l}$ and $z_{r}$ denote rule-ordered counterparts as:

$z_{l}=\left(z_{l}^{1}, \ldots, z_{l}^{M}\right)^{T}$ and $z_{r}=\left(z_{r}^{1}, \ldots, z_{r}^{M}\right)^{T}$

$z_{l}=\frac{\sum_{i=1}^{L} z_{l}^{i} \vec{a}^{i}+\sum_{i=L+1}^{M} z_{l}^{i} \underline{a}^{i}}{\sum_{i=1}^{L} \bar{a}^{i}+\sum_{i=L+1}^{M} \underline{a}^{i}}, z_{r}=\frac{\sum_{i=1}^{R} z_{r}^{i} \vec{a}^{i}+\sum_{i=R+1}^{M} z_{r}^{i} \underline{a}^{i}}{\sum_{i=1}^{R} \bar{a}^{i}+\sum_{i=R+1}^{M} \underline{a}^{i}}$,

in which $y_{l}=Q_{l} z_{l}$ and $y_{r}=Q_{r} z_{r}$, where $Q_{l}$ and $Q_{r}$ are an $M \times M$ permutation matrix.

Now, we should be going from the rule-unordered versions to the rule-ordered versions [13], [14]. For re-express $y_{r}$ in terms of rule-ordered quantities, we have to re-express the four sums of $y_{r}$ in (39) as the follows:

Let $\underline{\mathbf{b}}=\left(\underline{b}^{1}, \underline{b}^{2}, \ldots, \underline{\underline{b}}^{M}\right)^{T} \equiv Q_{r} \underline{\mathbf{a}}$ and $\overline{\mathbf{b}}=\left(\bar{b}^{1}, \bar{b}^{2}, \ldots, \bar{b}^{M}\right)^{T} \equiv Q_{r} \cdot \overline{\mathbf{a}}$,

$\mathbf{E}_{\mathbf{1 1}}=\left(e_{1}\left|e_{2}\right| \ldots\left|e_{R}\right| 0|\ldots| 0\right)_{R \times M}^{T}$, where $e_{i}=R \times 1$ is the $i^{t h}$ elementary vector, and

$\mathbf{E}_{22}=\left(0|\ldots| 0\left|\varepsilon_{1}\right| \varepsilon_{2}|\ldots| \varepsilon_{M-R}\right)_{(M-R) \times 1}^{T}$, where

$\varepsilon_{i}=(M-R) \times 1$ is the $i^{t h}$ elementary vector, therefore

$\sum_{i=1}^{R} \underline{b}^{i} y_{r}^{i}=$

$\left(e_{1}\left|e_{2}\right| \ldots\left|e_{R}\right| 0|\ldots| 0\right)^{T}\left(\underline{b}^{1}, \underline{b}^{2}, \ldots, \underline{b}^{M}\right)^{T}\left(e_{1}\left|e_{2}\right| \ldots\left|e_{R}\right| 0|\ldots| 0\right)\left(y_{r}^{1}, \ldots, y_{r}^{M}\right)$

$$
\begin{aligned}
& =\left(\mathbf{E}_{\mathbf{1 1}} \underline{\mathbf{b}}\right)^{T}\left(\mathbf{E}_{\mathbf{1 1}} y_{r}\right)=\left(\mathbf{E}_{\mathbf{1 1}} Q_{r} \cdot \underline{\mathbf{a}}\right)^{T}\left(\mathbf{E}_{\mathbf{1 1}} Q_{r} \cdot z_{r}\right) \\
& =\underline{\mathbf{a}}^{T} \underbrace{Q_{r}{ }^{T} \mathbf{E}_{\mathbf{1 1}}{ }^{T} \mathbf{E}_{\mathbf{1 1}} Q_{r}} z_{r}=\underline{\mathbf{a}}^{T} \underbrace{H_{r 1} z_{r}}=\underline{\mathbf{a}}^{T} \sigma_{r} .
\end{aligned}
$$

$\sum_{i=R+1}^{M} \bar{b}^{i} y_{r}^{i}=\left(0|\ldots| 0\left|\varepsilon_{1}\right| \varepsilon_{2}|\ldots| \varepsilon_{M-R}\right)^{T}\left(\bar{b}^{1}, \bar{b}^{2}, \ldots, \bar{b}^{M}\right)^{T} *$

$$
\begin{aligned}
& \left(0|\ldots| 0\left|\varepsilon_{1}\right| \varepsilon_{2}|\ldots| \varepsilon_{M-R}\right)\left(y_{r}^{1}, \ldots, y_{r}^{M}\right) \\
= & \left(\mathbf{E}_{\mathbf{2 2}} \overline{\mathbf{b}}\right)^{T}\left(\mathbf{E}_{\mathbf{2 2}} y_{l}\right)=\left(\mathbf{E}_{\mathbf{2 2}} Q_{r} \cdot \overline{\mathbf{a}}\right)^{T}\left(\mathbf{E}_{\mathbf{2 2}} Q_{r} \cdot z_{r}\right) \\
= & \overline{\mathbf{a}}^{T} \underbrace{Q_{r}{ }^{T} \mathbf{E}_{\mathbf{2 2}}{ }^{T} \mathbf{E}_{\mathbf{2 2}} Q_{r}} z_{r}=\overline{\mathbf{a}}^{T} \underbrace{H_{r 2} z_{r}}=\overline{\mathbf{a}}^{T} \rho_{r}
\end{aligned}
$$

in which $\left(Q_{r}{ }^{T} \mathbf{E}_{\mathbf{1 1}}{ }^{T} \mathbf{E}_{\mathbf{1 1}} Q_{r}\right)_{M \times M}=H_{r 1}, \quad\left(H_{r 1} z_{r}\right)_{M \times 1}=\sigma_{r}$,

$\left(Q_{r}{ }^{T} \mathbf{E}_{\mathbf{2 2}}{ }^{T} \mathbf{E}_{22} Q_{r}\right)_{M \times M}=H_{r 2}$, and $\left(H_{r 2} z_{r}\right)_{M \times 1}=\rho_{r}$

$$
\operatorname{suppose}(\underbrace{1,1, \ldots, 1}_{R}, 0, \ldots, 0)_{M \times 1}=k_{1 r},(0, \ldots, 0, \underbrace{1,1, \ldots, 1}_{M-R})_{M \times 1}=k_{2 r}
$$$$
\sum_{i=1}^{R} \underline{b}^{i}=(1,1, \ldots, 1,0, \ldots, 0)^{T}\left(\underline{b}^{1}, \underline{b}^{2}, \ldots, \underline{b}^{M}\right)=\underbrace{k_{1 r}{ }^{T} Q_{r}} \cdot \underline{\mathbf{a}}
$$$$
=\tau_{r}^{T} \underline{\mathbf{a}}
$$

$\sum_{i=L+1}^{M} \bar{b}^{i}=(0, \ldots, 0,1,1, \ldots, 1)^{T}\left(\bar{b}^{1}, \bar{b}^{2}, \ldots, \bar{b}^{M}\right)$

$$
=\underbrace{k_{2 r}{ }^{T} Q_{r}} \cdot \overline{\mathbf{a}}=\vartheta_{r}^{T} \overline{\mathbf{a}}
$$

in which $\left(k_{1 r}{ }^{T} Q_{r}\right)_{1 \times M}=\tau_{r}$ and $\left(k_{2 r}{ }^{T} Q_{r}\right)_{1 \times M}=\vartheta_{r}$.
Consequently, from (41)-(44), we obtained:

$$
\begin{aligned}
y_{r} & =\frac{\underline{\mathbf{a}}^{T} H_{r 1} z_{r}+\overline{\mathbf{a}}^{T} H_{r 2} z_{r}}{k_{1 r}{ }^{T} Q_{r} \underline{\mathbf{a}}+k_{2 r}{ }^{T} Q_{r} \overline{\mathbf{a}}} \\
& =\frac{\underline{\mathbf{a}}^{T} \boldsymbol{\sigma}_{r}+\overline{\mathbf{a}}^{T} \boldsymbol{\rho}_{r}}{\boldsymbol{\tau}_{r}^{T} \underline{\mathbf{a}}+\boldsymbol{\vartheta}_{r}^{T} \overline{\mathbf{a}}}=\frac{\underline{\mathbf{a}}^{T} \boldsymbol{\sigma}_{r}+\overline{\mathbf{a}}^{T} \boldsymbol{\rho}_{r}}{\underline{\mathbf{a}}^{T} \boldsymbol{\tau}_{r}+\overline{\mathbf{a}}^{T} \boldsymbol{\vartheta}_{r}} \\
& =\frac{\sum_{i=1}^{M} \sigma_{r, i} \underline{b}^{i}+\sum_{i=1}^{M} \rho_{r, i} \bar{b}^{i}}{\sum_{i=1}^{M} \tau_{r, i} \underline{b}^{i}+\sum_{i=1}^{M} \vartheta_{r, i} \bar{b}^{i}}
\end{aligned}
$$

Note that Equation (45a) includes the entire $\underline{\mathbf{a}}$, $\overline{\mathbf{a}}$ and $z_{r}$ vectors. The matrices $H_{r 1}, H_{r 2}$ and the vectors $k_{1 r}, k_{2 r}$ will automatically get out of the unnecessary elements of $\underline{\mathbf{a}}$ and $\overline{\mathbf{a}}$ those depend on $R$. Similarly, $y_{l}$ can be express in terms of rule-ordered quantities, [15], [17].

\section{COMPUTATION of $\partial \cosh (\alpha) / \partial \theta_{i, k}^{l}$ for ANTECEDENT and CONSEQU- ENT PARAMETERS}

In this Section, we can built mathematical formulas to calculate the derivatives $\partial \cosh (\alpha) / \partial \theta$ first for antecedent parameters, and second for consequent parameters as follows [13]:

\subsection{Computation of $\partial \cosh (\alpha) / \partial \theta_{i, k}^{l}$ for Parameters of Antecedent}

Parameters of an antecedent are the parameters that describe antecedent MFs [12]. Let us denote any one of the antecedent parameters that will be tuned as $\theta_{i, k}^{l}(i=1, \ldots, n$ and $l=$ $1, \ldots, M)$, and $m$ denotes the number of parameters when there can be more than one parameter related with the MF of each antecedent $i$ and rule $l$. From Equation (35), where $f_{n s}(x)$ is given as [Mitchell 2006]:

$f_{n s}(x)=\frac{\left(y_{l}(x)+y_{r}(x)\right)}{2}$,

Therefore,

$$
\begin{aligned}
\frac{\partial \cosh (\alpha)}{\partial \theta_{i, k}^{l}} & =\frac{1}{2}\left[\frac{\partial\left(e^{(\alpha)}\right)^{-1}}{\partial \theta_{i, k}^{l}}+\frac{\partial\left(e^{(\alpha)}\right)}{\partial \theta_{i, k}^{l}}\right] \\
& =\frac{1}{2}\left[\frac{\partial\left(e^{(\alpha)}\right)^{-1}}{\partial f_{n s}} \cdot \frac{\partial f_{n s}}{\partial \theta_{i, k}^{l}}+\frac{\partial\left(e^{(\alpha)}\right)}{\partial f_{n s}} \cdot \frac{\partial f_{n s}}{\partial \theta_{i, k}^{l}}\right] \\
& =\frac{1}{2}\left[\begin{array}{l}
\frac{\partial\left(e^{(\alpha)}\right)^{-1}}{\partial f_{n s}} \cdot\left(\frac{\partial f_{n s}}{\partial y_{l}} \cdot \frac{\partial y_{l}}{\partial \theta_{i, k}^{l}}+\frac{\partial f_{n s}}{\partial y_{r}} \cdot \frac{\partial y_{r}}{\partial \theta_{i, k}^{l}}\right) \\
+\frac{\partial\left(e^{(\alpha)}\right)}{\partial f_{n s}} \cdot\left(\frac{\partial f_{n s}}{\partial y_{l}} \cdot \frac{\partial y_{l}}{\partial \theta_{i, k}^{l}}+\frac{\partial f_{n s}}{\partial y_{r}} \cdot \frac{\partial y_{r}}{\partial \theta_{i, k}^{l}}\right)
\end{array}\right] \\
& =\frac{1}{2}\left[\begin{array}{l}
\left(\frac{\partial\left(e^{(\alpha)}\right)^{-1}}{\partial f_{n s}}+\frac{\partial\left(e^{(\alpha)}\right)}{\partial f_{n s}}\right) * \\
\left(\frac{\partial f_{n s}}{\partial y_{l}} \cdot \frac{\partial y_{l}}{\partial \theta_{i, k}^{l}}+\frac{\partial f_{n s}}{\partial y_{r}} \cdot \frac{\partial y_{r}}{\partial \theta_{i, k}^{l}}\right)
\end{array}\right]
\end{aligned}
$$

Since, $\partial f_{n s}(x) / \partial y_{l}(x)=\partial f_{n s}(x) / \partial y_{r}(x)=1 / 2$ and

$$
\frac{\partial\left(e^{(\alpha)}\right)^{-1}}{\partial f_{n s}}+\frac{\partial\left(e^{(\alpha)}\right)}{\partial f_{n s}}=\left(\begin{array}{c}
\frac{-4}{\left[f_{n s}\left(x^{(\alpha)}\right)-y^{(\alpha)}\right]^{3}} \\
+\left[f_{n s}\left(x^{(\alpha)}\right)-y^{(\alpha)}\right]^{2}
\end{array}\right)
$$

Then, from (47) and (48) we obtain, 
$\frac{\partial \cosh (\alpha)}{\partial \theta_{i, k}^{l}}=\left[\left(\begin{array}{l}\frac{\left[f_{n s}\left(x^{(\alpha)}\right)-y^{(\alpha)}\right]}{4} \\ -\frac{1}{\left[f_{n s}\left(x^{(\alpha)}\right)-y^{(\alpha)}\right]^{3}}\end{array}\right) \cdot\left(\frac{\partial y_{l}}{\partial \theta_{i, k}^{l}}+\frac{\partial y_{r}}{\partial \theta_{i, k}^{l}}\right)\right]$

Now handle $y_{l}$ and $y_{r}$ in (49) such as functions of $\overline{\mathbf{a}}$ and $\underline{\mathbf{a}}$; therefore,

$\frac{\partial y_{r}}{\partial \theta_{i, k}^{l}}=\sum_{j=1}^{M} \frac{\partial y_{r}}{\partial \underline{a}^{j}} \cdot \frac{\partial \underline{a}^{j}}{\partial \theta_{i, k}^{l}}+\frac{\partial y_{r}}{\partial \bar{a}^{j}} \cdot \frac{\partial \bar{a}^{j}}{\partial \theta_{i, k}^{l}}$,

$\frac{\partial y_{l}}{\partial \theta_{i, k}^{l}}=\sum_{j=1}^{M} \frac{\partial y_{l}}{\partial \bar{a}^{j}} \cdot \frac{\partial \bar{a}^{j}}{\partial \theta_{i, k}^{l}}+\frac{\partial y_{l}}{\partial \underline{a}^{j}} \cdot \frac{\partial \underline{a}^{j}}{\partial \theta_{i, k}^{l}}$.

Consequently, we must estimate all of the derivatives in (50) and (51). From the formula of $y_{r}$ in (45), we obtain

$\frac{\partial y_{r}}{\partial \underline{a}^{i}}=\frac{\sigma_{r, i}\left(\underline{\mathbf{a}}^{T} \boldsymbol{\tau}_{r}+\overline{\mathbf{a}}^{T} \boldsymbol{\vartheta}_{r}\right)-\tau_{r, i}\left(\underline{\mathbf{a}}^{T} \boldsymbol{\sigma}_{r}+\overline{\mathbf{a}}^{T} \boldsymbol{\rho}_{r}\right)}{\left(\underline{\mathbf{a}}^{T} \boldsymbol{\tau}_{r}+\overline{\mathbf{a}}^{T} \boldsymbol{\vartheta}_{r}\right)^{2}}$,

so that

$$
\begin{aligned}
\frac{\partial y_{r}}{\partial \underline{a}^{i}} & =\frac{\sigma_{r, i}}{\left(\underline{\mathbf{a}}^{T} \boldsymbol{\tau}_{r}+\overline{\mathbf{a}}^{T} \boldsymbol{\vartheta}_{r}\right)}-\frac{y_{r} \tau_{r, i}}{\left(\underline{\mathbf{a}}^{T} \boldsymbol{\tau}_{r}+\overline{\mathbf{a}}^{T} \boldsymbol{\vartheta}_{r}\right)} \\
& =\frac{\sigma_{r, i}-y_{r} \tau_{r, i}}{\left(\underline{\mathbf{a}}^{T} \boldsymbol{\tau}_{r}+\overline{\mathbf{a}}^{T} \boldsymbol{\vartheta}_{r}\right)}
\end{aligned}
$$

From (45) and (A-5) with the same manner, we have obtained

$\frac{\partial y_{r}}{\partial \bar{a}^{i}}=\frac{\rho_{r, i}-y_{r} \vartheta_{r, i}}{\left(\underline{\mathbf{a}}^{T} \boldsymbol{\tau}_{r}+\overline{\mathbf{a}}^{T} \boldsymbol{\vartheta}_{r}\right)}$

$\frac{\partial y_{l}}{\partial \underline{a}^{i}}=\frac{\rho_{l, i}-y_{l} \vartheta_{l, i}}{\left(\overline{\mathbf{a}}^{T} \boldsymbol{\tau}_{l}+\underline{\mathbf{a}}^{T} \boldsymbol{\vartheta}_{l}\right)}$

$\frac{\partial y_{l}}{\partial \bar{a}^{i}}=\frac{\sigma_{l, i}-y_{l} \tau_{l, i}}{\left(\overline{\mathbf{a}}^{T} \boldsymbol{\tau}_{l}+\underline{\mathbf{a}}^{T} \boldsymbol{\vartheta}_{l}\right)}$

In order to obtain $\partial y_{r} / \partial \theta_{i, k}^{l}$, calculate $\partial \underline{a}^{l} / \partial \theta_{i, k}^{l}$ and $\partial \bar{a}^{l} / \partial \theta_{i, k}^{l}$. Now, we just need to calculate $\left[\underline{a}^{l}, \bar{a}^{l}\right]$, then try to choose an operator t-norm and create the functions $\underline{\mu}_{\hat{o}_{i}^{l}}\left(x_{i}\right)$ and $\bar{\mu}_{\hat{o}_{i}^{l}}\left(x_{i}\right)$, in which,

$\underline{\mu}_{\hat{O}_{i}^{l}}\left(x_{i}\right)=\int_{x_{i} \in X_{i}}\left[\underline{\mu}_{\hat{X}_{i}}\left(x_{i}\right) * \underline{\mu}_{\hat{A}_{i}^{l}}\left(x_{i}\right)\right] / x_{i}$

$\bar{\mu}_{\hat{o}_{i}^{l}}\left(x_{i}\right)=\int_{x_{i} \in X_{i}}\left[\bar{\mu}_{\hat{X}_{i}}\left(x_{i}\right) * \bar{\mu}_{\hat{A}_{i}^{l}}\left(x_{i}\right)\right] / x_{i}$

Further, calculate the values of $x_{i}$ that are related with $\left(\sup _{x_{i}}{\underline{\mu_{\hat{O}_{i}^{l}}}}\left(x_{i}\right)\right)$ and $\left(\sup _{x_{i}} \bar{\mu}_{\hat{O}_{i}^{l}}\left(x_{i}\right)\right)$ as follows:

$\underline{x}_{i, s}^{l}=\sup _{x_{i}} \underline{\mu}_{\hat{o}_{i}^{l}}\left(x_{i}\right)$ And $\bar{x}_{i, s}^{l}=\sup _{x_{i}} \bar{\mu}_{\hat{O}_{i}^{l}}\left(x_{i}\right)$,

Where $\underline{x}_{i, s}^{l}$ and $\bar{x}_{i, s}^{l}$ are the maximum values of $x_{i}$, then we have estimated $\underline{a}_{i}^{l}\left(x_{i}{ }^{\prime}\right)$ and $\bar{a}_{i}^{l}\left(x_{i}{ }^{\prime}\right)$, in which

$\underline{a}_{i}^{l}\left(x_{i}^{\prime}\right)=\underline{\mu}_{\hat{O}_{i}^{l}}\left(\underline{x}_{i, s}^{l}\right)$,

And

$\bar{a}_{i}^{l}\left(x_{i}^{\prime}\right)=\bar{\mu}_{\hat{o}_{i}^{l}}\left(\bar{x}_{i, s}^{l}\right)$.

Observe that $\underline{x}_{i, s}^{l}$ and $\bar{x}_{i, s}^{l}$ are depended on measurement $x_{i}^{\prime}$, and computed

$\begin{aligned} \underline{a}^{l}\left(x^{\prime}\right) & =T_{i=1}^{n} \underline{a}_{i}^{l}\left(x_{i}^{\prime}\right)=T_{i=1}^{n} \underline{\mu}_{\hat{\sigma}_{i}^{l}}\left(\underline{x}_{i, s}^{l}\right) \\ & =\mu_{\hat{O}_{1}^{l}}\left(\underline{x}_{1, s}^{l}\right) * \ldots * \mu_{\hat{O}_{n}^{l}}\left(\underline{x}_{n, s}^{l}\right)\end{aligned}$ and

$$
\begin{aligned}
\bar{a}^{l}\left(x^{\prime}\right) & =T_{i=1}^{n} \bar{a}_{i}^{l}\left(x_{i}^{\prime}\right)=T_{i=1}^{n} \bar{\mu}_{\hat{O}_{i}^{l}}\left(\bar{x}_{i, s}^{l}\right) \\
& =\bar{\mu}_{\hat{O}_{1}^{l}}\left(\bar{x}_{1, s}^{l}\right) * \ldots * \bar{\mu}_{\hat{o}_{n}^{l}}\left(\bar{x}_{n, s}^{l}\right) .
\end{aligned}
$$

The major difference between calculating the firing interval for an IT2 non-singleton T2 FLS and an interval singleton T2 FLS is to calculate $\underline{x}_{i, s}^{l}$ and $\bar{x}_{i, s}^{l}$ [23], [26]. Because no parameters are shared across rules or MFs, then for specified values of $i$ and $l$. Parameters of antecedent $\theta_{i, k}^{l}$ can appear in

$\underline{\mu}_{\hat{o}_{i}^{l}}\left(\underline{x}_{i, S}^{l}\right)$ and $\quad \bar{\mu}_{\hat{o}_{i}^{l}}\left(\bar{x}_{i, s}^{l}\right)$ and cannot appear in $\underline{\mu}_{\hat{o}_{j}^{l}}\left(\underline{x}_{j, s}^{l}\right)$ and $\bar{\mu}_{\hat{o}_{j}^{l}}\left(\bar{x}_{j, s}^{l}\right)$ for $\mathrm{j} \neq \mathrm{i}$, that means,

$\frac{\partial \underline{a}^{j}}{\partial \theta_{i, k}^{l}}=\left\{\begin{array}{ll}\frac{\partial \underline{a}^{l}}{\partial \theta_{i, k}^{l}} & \forall j \neq l \\ 0 & \forall j \neq l\end{array} \quad\right.$ and

$\frac{\partial \bar{a}^{j}}{\partial \theta_{i, k}^{l}}= \begin{cases}\frac{\partial \bar{a}^{l}}{\partial \theta_{i, k}^{l}} & \forall j \neq l \\ 0 & \forall j \neq l\end{cases}$

Therefore, from (62), simplify (50) and (51) as the following:

$\frac{\partial y_{r}}{\partial \theta_{i, k}^{l}}=\frac{\partial y_{r}}{\partial \underline{a}^{l}} \cdot \frac{\partial \underline{a}^{l}}{\partial \theta_{i, k}^{l}}+\frac{\partial y_{r}}{\partial \bar{a}^{l}} \cdot \frac{\partial \bar{a}^{l}}{\partial \theta_{i, k}^{l}}$
$\frac{\partial y_{l}}{\partial \theta_{i, k}^{l}}=\frac{\partial y_{l}}{\partial \bar{a}^{l}} \cdot \frac{\partial \bar{a}^{l}}{\partial \theta_{i, k}^{l}}+\frac{\partial y_{l}}{\partial \underline{a}^{l}} \cdot \frac{\partial \underline{a}^{l}}{\partial \theta_{i, k}^{l}}$

Thus, the last part in (49) become:

$$
\begin{aligned}
\frac{\partial y_{l}}{\partial \theta_{i, k}^{l}}+\frac{\partial y_{r}}{\partial \theta_{i, k}^{l}} & = \\
& =\left(\frac{\partial y_{l}}{\partial \bar{a}} \cdot \frac{\partial \bar{a}^{l}}{\partial \theta_{i, k}^{l}}+\frac{\partial y_{l}}{\partial \underline{a}^{l}} \cdot \frac{\partial \underline{a}^{l}}{\partial \theta_{i, k}^{l}}\right)+\left(\frac{\partial y_{r}}{\partial \underline{a}^{l}} \cdot \frac{\partial \underline{a}^{l}}{\partial \theta_{i, k}^{l}}+\frac{\partial y_{r}}{\partial \bar{a}^{l}} \cdot \frac{\partial \bar{a}^{l}}{\partial \theta_{i, k}^{l}}\right) \\
& =\left(\frac{\partial y_{l}}{\partial \bar{a}^{l}}+\frac{\partial y_{r}}{\partial \bar{a}^{l}}\right) \frac{\partial \bar{a}^{l}}{\partial \theta_{i, k}^{l}}+\left(\frac{\partial y_{l}}{\partial \underline{a}^{l}}+\frac{\partial y_{r}}{\partial \underline{a}^{l}}\right) \frac{\partial \underline{a}^{l}}{\partial \theta_{i, k}^{l}}
\end{aligned}
$$

Consequently, from (49) and (64), we obtained,

$$
\frac{\partial \cosh (\alpha)}{\partial \theta_{i, k}^{l}}=\left[\begin{array}{l}
\left(\begin{array}{c}
\frac{\left[f_{n s}\left(x^{(\alpha)}\right)-y^{(\alpha)}\right]}{4} \\
-\frac{1}{\left[f_{n s}\left(x^{(\alpha)}\right)-y^{(\alpha)}\right]^{3}}
\end{array}\right) * \\
\left(\begin{array}{c}
\left(\frac{\partial y_{l}}{\partial \bar{a}^{l}}+\frac{\partial y_{r}}{\partial \bar{a}^{l}}\right) \frac{\partial \bar{a}^{l}}{\partial \theta_{i, k}^{l}} \\
+\left(\frac{\partial y_{l}}{\partial \underline{a}^{l}}+\frac{\partial y_{r}}{\partial \underline{a}^{l}}\right) \frac{\partial \underline{a}^{l}}{\partial \theta_{i, k}^{l}}
\end{array}\right)
\end{array}\right]
$$

Now, since the parameters of antecedent $\theta_{i, k}^{l}$ can appear in $\underline{\mu}_{\hat{o}_{i}^{l}}\left(\underline{x}_{i, s}^{l}\right)$ and $\bar{\mu}_{\hat{o}_{i}^{l}}\left(\bar{x}_{i, s}^{l}\right)$ and cannot appear in $\underline{\mu}_{\hat{\sigma}_{j}^{l}}\left(\underline{x}_{j, s}^{l}\right)$ and $\bar{\mu}_{\hat{O}_{j}^{l}}\left(\bar{x}_{j, s}^{l}\right)$ for $j \neq i$ with Equations (60) and (61) [27], and we obtain for t-norm,

$$
\begin{aligned}
& \frac{\partial \underline{a}^{l}\left(x_{i}^{(\alpha)}\right)}{\partial \theta_{i, k}^{l}}=\left(T_{j=1}^{n} \underline{\mu}_{\hat{o}_{j}^{l}}\left(\underline{x}_{j, s}^{l}\right)\right) \times \frac{\partial \underline{\mu}_{\hat{o}_{i}^{l}}\left(\underline{x}_{i, s}^{l}\right)}{\partial \theta_{i, k}^{l}} \\
& \frac{\partial \bar{a}^{l}\left(x_{i}^{(\alpha)}\right)}{\partial \theta_{i, k}^{l}}=\left(T_{\substack{j=1 \\
j \neq i}}^{n} \bar{\mu}_{\hat{o}_{j}^{l}}\left(\bar{x}_{j, s}^{l}\right)\right) \times \frac{\partial \bar{\mu}_{\hat{o}_{i}^{l}}\left(\bar{x}_{i, s}^{l}\right)}{\partial \theta_{i, k}^{l}}
\end{aligned}
$$


Next, the remaining computation of $\partial \underline{\mu}_{\hat{o}_{i}^{l}}\left(\underline{x}_{i, s}^{l}\right) / \partial \theta_{i, k}^{l}$ and $\partial \bar{\mu}_{\hat{O}_{i}^{l}}\left(\bar{x}_{i, s}^{l}\right) / \partial \theta_{i, k}^{l}$ need designation of antecedent MFs and their related FOUs, therefore, we can be summarized the steps for calculation $\partial \cosh (\alpha) / \partial \theta_{i, k}^{l}$ as follows:

\subsection{Algorithm (The Derivatives for Antecedent Parameters to Calculate $\left.\partial \cosh (\alpha) / \partial \theta_{i, k}^{l}\right)$}

Step 1: For input vector $x^{(\alpha)}$, specify active states for all $\mathrm{i}=1, \ldots, \mathrm{n}$.

Step 2: For all $\mathrm{i}=1, \ldots, \mathrm{n}$ and for all $l=1, \ldots, M$, using (58)(59) calculate $\underline{x}_{i, s}^{l}, \bar{x}_{i, s}^{l}, \underline{\mu}_{\widehat{O}_{i}^{l}}\left(\underline{x}_{i, s}^{l}\right)$ and $\bar{\mu}_{\widehat{O}_{i}^{1}}\left(\bar{x}_{i, s}^{l}\right)$.

Step 3: For all $l=1, \ldots, M$,

a) Using (61) to calculate $\underline{a}^{l}\left(x^{(\alpha)}\right)$, thus depend on $\theta_{i, k}^{l}$ and $\partial \mu_{\hat{o}_{i}^{l}}\left(\underline{x}_{i, s}^{l}\right) / \partial \theta_{i, k}^{l} \quad$ with $\quad$ (67), calculate $\partial \underline{a}^{l}\left(x^{(\alpha)}\right) / \partial \theta_{i, k}^{l}$

b) Using (62) to calculate $\bar{a}^{l}\left(x^{(\alpha)}\right)$, thus depend on $\theta_{i, k}^{l}$ and $\partial \bar{\mu}_{\hat{O}_{i}^{l}}\left(\bar{x}_{i, s}^{l}\right) / \partial \theta_{i, k}^{l} \quad$ with $\quad$ (68), calculate $\partial \bar{a}^{l}\left(x^{(\alpha)}\right) / \partial \theta_{i, k}^{l}$

Step 4: Using the KM iterative procedure

a) Depending on $z_{l}^{i}$, calculate $y_{l}, L$ and $Q_{l}$.

b) Depending on $z_{r}^{i}$, calculate $y_{r}, R$ and $Q_{r}$

From (a) and (b) with (46), we can calculate $f_{n s}\left(x^{(\alpha)}\right)$, and thus, calculate the first term in (65),

$\left(\frac{\left[f_{n s}\left(x^{(\alpha)}\right)-y^{(\alpha)}\right]}{4}-\frac{1}{\left[f_{n s}\left(x^{(\alpha)}\right)-y^{(\alpha)}\right]^{3}}\right)$.

Step 5: For $\underline{\boldsymbol{a}}=\left(\underline{a}^{1}, \underline{a}^{2}, \ldots, \underline{a}^{M}\right)^{T}$ and $\overline{\boldsymbol{a}}=\left(\bar{a}^{1}, \bar{a}^{2}, \ldots, \bar{a}^{M}\right)^{T}$

a) Using definitions of $\boldsymbol{E}_{\mathbf{1}}$ and $\boldsymbol{E}_{\mathbf{2}}$ in order to calculate $H_{l 1}, H_{l 2}, k_{1 l}, k_{2 l}, \boldsymbol{\sigma}_{l}, \boldsymbol{\rho}_{l}, \boldsymbol{\tau}_{l}^{T}$ and $\boldsymbol{\vartheta}_{l}^{T}$.

b) Using definitions of $\mathbf{E}_{\mathbf{1 1}}$ and $\mathbf{E}_{\mathbf{2}} 2$ in order to calculate $H_{r 1}, H_{r 2}, k_{1 r}, k_{2 r}, \boldsymbol{\sigma}_{r}, \boldsymbol{\rho}_{r}, \boldsymbol{\tau}_{r}^{T}$ and $\boldsymbol{\vartheta}_{r}^{T}$.

\section{Step 6:}

a) Using (54) and (55) to calculate $\partial \mathrm{y}_{\mathrm{l}} / \partial \underline{\mathrm{a}}^{\mathrm{i}}$ and $\partial \mathrm{y}_{\mathrm{l}} / \partial \overline{\mathrm{a}}^{\mathrm{i}}$, respectively.

b) Using (52) and (53) to calculate $\partial \mathrm{y}_{\mathrm{r}} / \partial \underline{a}^{\mathrm{i}}$ and $\partial \mathrm{y}_{\mathrm{r}} / \partial \overline{\mathrm{a}}^{\mathrm{i}}$, respectively.

Step 7: From the results of step 3 and step 4 with using (66), we can be calculated $\partial \cosh (\alpha) / \partial \theta_{i, k}^{l}$.

\subsection{Computation of $\partial \cosh (\alpha) / \partial \theta^{i}$ for Parameters of Consequent}

Parameters of consequent are the parameters that describe consequent MFs. When, we use height type-reduction, then those parameters can be replaced by the two end-points of the T2 consequent sets [28], [25], and this can reduce the number of model parameters [3], [18]. Observe that the parameters of consequent do not need the " $i$ " and " $k$ " subscript symbols in $\theta_{i, k}^{j}$.
Since, $\theta^{j}=z_{l}^{j}$ or $\theta^{j}=z_{r}^{j}$ and from (36) and (46), we obtain,

$$
\begin{aligned}
& \frac{\partial \cosh (\alpha)}{\partial z_{l}^{j}}=\frac{\partial \cosh (\alpha)}{\partial f_{n s}\left(x^{(\alpha)}\right)} \cdot \frac{\partial f_{n s}\left(x^{(\alpha)}\right)}{\partial y_{l}} \cdot \frac{\partial y_{l}}{\partial z_{l}^{j}} \\
& =\left(\frac{\left[f_{n s}\left(x^{(\alpha)}\right)-y^{(\alpha)}\right]}{4}-\frac{1}{\left[f_{n s}\left(x^{(\alpha)}\right)-y^{(\alpha)}\right]^{3}}\right) \cdot \frac{\partial y_{l}}{\partial z_{l}^{j}},
\end{aligned}
$$

and

$$
\begin{aligned}
& \frac{\partial \cosh (\alpha)}{\partial z_{r}^{j}}= \\
& \left(\frac{\left[f_{n s}\left(x^{(\alpha)}\right)-y^{(\alpha)}\right]}{4}-\frac{1}{\left[f_{n s}\left(x^{(\alpha)}\right)-y^{(\alpha)}\right]^{3}}\right) \cdot \frac{\partial y_{r}}{\partial z_{r}^{j}} .
\end{aligned}
$$

From (45a) and (A-5) with the fact $\frac{\partial}{\partial z}\left(\beta^{T} z\right)=\beta$, we obtain,

$\frac{\partial y_{l}}{\partial z_{l}}=\left(\frac{\underline{\boldsymbol{a}}^{T} H_{l 1}+\overline{\boldsymbol{a}}^{T} H_{l 2}}{k_{1 l}{ }^{T} Q_{l} \underline{\boldsymbol{a}}+k_{2 l}{ }^{T} Q_{l} \overline{\boldsymbol{a}}}\right)^{T}=\frac{H_{l 1}^{T} \underline{\underline{\boldsymbol{a}}}+H_{l 2}^{T} \overline{\boldsymbol{a}}}{k_{1 l}^{T} Q_{l} \underline{\boldsymbol{a}}+k_{2 l}^{T} Q_{l} \overline{\boldsymbol{a}}}$

$\frac{\partial y_{r}}{\partial z_{r}}=\left(\frac{\underline{\boldsymbol{a}}^{T} H_{r 1}+\overline{\boldsymbol{a}}^{T} H_{r 2}}{k_{1 r}{ }^{T} Q_{r} \underline{\boldsymbol{a}}+k_{2 r}{ }^{T} Q_{r} \overline{\overline{\boldsymbol{a}}}}\right)^{T}=\frac{H_{r 1}^{T}{ }^{T} \underline{\boldsymbol{a}}+H_{r 2}^{T} \overline{\boldsymbol{a}}}{k_{1 r}^{T} Q_{r} \underline{\boldsymbol{a}}+k_{2 r}^{T} Q_{r} \overline{\boldsymbol{a}}}(72)$

Since, $\frac{\partial y_{l}}{\partial z_{l}}=\left[\frac{\partial y_{l}}{\partial z_{l}^{1}}, \frac{\partial y_{l}}{\partial z_{l}^{1}}, \ldots, \frac{\partial y_{l}}{\partial z_{l}^{1}}\right]^{T}$, so that $e_{j}^{T} \cdot \frac{\partial y_{l}}{\partial z_{l}}=\frac{\partial y_{l}}{\partial z_{l}^{j}}$. Consequently, re-write the Equations (71) and (72) as the following:

$\frac{\partial y_{l}}{\partial z_{l}^{j}}=e_{j}^{T}\left(\frac{H_{l 1}^{T^{T}} \underline{\boldsymbol{a}}+H_{l 2}^{T} \overline{\boldsymbol{a}}}{k_{1 l}^{T} Q_{l} \underline{\boldsymbol{a}}+k_{2 l}^{T} Q_{l} \overline{\bar{a}}}\right)$
$\frac{\partial y_{r}}{\partial z_{r}^{j}}=e_{j}^{T}\left(\frac{H_{r 1}^{T} \underline{\boldsymbol{a}}+H_{r 2}^{T} \overline{\boldsymbol{a}}}{k_{1 r}^{T} Q_{r} \underline{\boldsymbol{a}}+k_{2 r}^{T} Q_{r} \overline{\boldsymbol{a}}}\right)$

This completes the derivations of derivative models for calculate $\partial \cosh (\alpha) / \partial \theta_{\mathrm{i}, \mathrm{k}}^{\mathrm{l}}$.

\section{APPLICATION}

Consider the case of Gaussian primary MF having an uncertain mean and standard deviation that take on values in $m \in\left[m_{1}, m_{2}\right]$, and $\sigma \in\left[\sigma_{1}, \sigma_{2}\right]$ respectively, as follows [19], [27]:

$\mu_{A}(x)=\exp \left(-\frac{(x-m)^{2}}{2 \sigma^{2}}\right), m \in\left[m_{1}, m_{2}\right], \sigma \in\left[\sigma_{1}, \sigma_{2}\right]$

For calculate $\partial \bar{a}^{l} / \partial \theta_{i, k}^{l}$ and $\partial \underline{a}^{l} / \partial \theta_{i, k}^{l}$ using (67) and (68), we need to calculate $\partial \underline{\mu}_{\hat{o}_{i}^{l}}\left(\underline{x}_{i, s}^{l}\right) / \partial \theta_{i, k}^{l}$ and $\partial \bar{\mu}_{\hat{o}_{i}^{l}}\left(\bar{x}_{i, s}^{l}\right) / \partial \theta_{i, k}^{l}$. Further, calculate $\partial \underline{\mu}_{\hat{o}_{i}^{l}}\left(\underline{x}_{i, s}^{l}\right) / \partial \theta_{i, k}^{l}$ and $\partial \bar{\mu}_{\hat{o}_{i}^{l}}\left(\bar{x}_{i, s}^{l}\right) / \partial \theta_{i, k}^{l}$ for antecedent Gaussian primary MFs with uncertain means and input measurement Gaussian primary MFs with uncertain standard deviations. Formulas for antecedent MFs and their lower and upper MFs are given as [13]:

$$
\begin{aligned}
& \mu_{i}^{l}(x)=\exp \left(-\frac{\left(x_{i}-m_{i}^{l}\right)^{2}}{2 \sigma_{i}^{l^{2}}}\right), m_{i}^{l} \in\left[m_{i, 1}^{l}, m_{i, 2}^{l}\right], \\
& i=1, \ldots, n, l=1, \ldots, M \text {. } \\
& \bar{\mu}_{i}^{l}\left(x_{i}\right)= \begin{cases}\exp \left(-\frac{\left(x_{i}-m_{i, 1}^{l}\right)^{2}}{2 \sigma_{i}^{l^{2}}}\right) & x_{i}<m_{i, 1}^{l} \\
1 & x_{i} \in\left[m_{i, 1}^{l}, m_{i, 2}^{l}\right] \\
\exp \left(-\frac{\left(x_{i}-m_{i, 2}^{l}\right)^{2}}{2 \sigma_{i}^{l^{2}}}\right) & m_{i, 2}^{l}<x_{i}\end{cases}
\end{aligned}
$$


$\underline{\mu}_{i}^{l}\left(x_{i}\right)= \begin{cases}\exp \left(-\frac{\left(x_{i}-m_{i, 2}^{l}\right)^{2}}{2 \sigma_{i}^{l^{2}}}\right) & x_{i} \leq \frac{m_{i, 1}^{l}+m_{i, 2}^{l}}{2} \\ \exp \left(-\frac{\left(x_{i}-m_{i, 1}^{l}\right)^{2}}{2 \sigma_{i}^{l^{2}}}\right) & \frac{m_{i, 1}^{l}+m_{i, 2}^{l}}{2}<x_{i}\end{cases}$

Formulas for input measurement MFs and their lower and upper MFs are:

$\mu_{i}^{l}\left(x_{i}\right)=\exp \left(-\frac{\left(x_{i}-x_{i}^{(j)}\right)^{2}}{2{\sigma_{i}^{l}}^{2}}\right), \quad \sigma_{i}^{l} \in\left[\sigma_{i 1}^{l}, \sigma_{i 2}^{l}\right]$,

$\bar{\mu}_{i}^{l}\left(x_{i}\right)=\exp \left(-\frac{\left(x_{i}-x_{i}^{(j)}\right)^{2}}{2 \sigma_{i, 2}^{l}{ }^{2}}\right), \underline{\mu}_{i}^{l}\left(x_{i}\right)=\exp \left(-\frac{\left(x_{i}-x_{i}^{(j)}\right)^{2}}{2 \sigma_{i, 1}^{l}{ }^{2}}\right)$

We have summarized the state of $x_{i}^{\prime}$ and the results for $\partial \underline{\mu}_{\hat{o}_{i}^{l}}\left(\underline{x}_{i, s}^{l}\right) / \partial \theta_{i, k}^{l}$ and $\partial \bar{\mu}_{\hat{o}_{i}^{l}}\left(\bar{x}_{i, s}^{l}\right) / \partial \theta_{i, k}^{l}$ that depend on, as a function of $x_{i}$, in Table II.

Suppose $\theta_{i, 1}^{l} \equiv m_{i 1}^{l}, \theta_{i, 2}^{l} \equiv m_{i 2}^{l}, \theta_{i, 3}^{l} \equiv \sigma_{i}^{l}, \theta_{i, 4}^{l} \equiv \sigma_{i 1}, \theta_{i, 5}^{l} \equiv$ $\sigma_{i 2}$. Depending on the formulas in Table I, we have calculate the exact derivatives of $\partial \underline{\mu}_{\hat{o}_{i}^{l}}\left(\underline{x}_{j, s}^{l}\right) / \partial \theta_{i k}^{l}$ and $\partial \bar{\mu}_{\hat{O}_{i}^{l}}\left(\bar{x}_{i, s}^{l}\right) / \partial \theta_{i, k}^{l}$. Tables II and III provide nonzero or zero derivatives of $\underline{\mu}_{\hat{o}_{i}^{l}}\left(\underline{x}_{i, s}^{l}\right)$ and $\bar{\mu}_{\hat{o}_{i}^{l}}\left(\bar{x}_{i, s}^{l}\right)$ with respect to all $\theta_{i, k}^{l}$ where $(k=1, \ldots, 5)$. The results in Table II have been provided $\underline{\mu}_{\hat{o}_{i}^{l}}\left(\underline{x}_{i, s}^{l}\right)$ which is needed to calculate $\partial \underline{a}^{l}\left(x_{i}^{(j)}\right) / \partial \theta_{i, k}^{l}$, and the results in Table III have been provided $\bar{\mu}_{\hat{O}_{i}^{l}}\left(\bar{x}_{i, s}^{l}\right)$ which is needed to calculate $\partial \bar{a}^{l}\left(x_{i}^{(j)}\right) / \partial \theta_{i, k}^{l}$. From the result of this application, note that $\underline{\mu}_{\hat{o}_{i}^{l}}\left(\underline{x}_{i, s}^{l}\right)$ depends on $\theta_{i, 4}^{l}=\sigma_{i 1}$ in all states, and $\bar{\mu}_{\hat{o}_{i}^{l}}\left(\bar{x}_{i, s}^{l}\right)$ depends on $\theta_{i, 5}^{l}=\sigma_{i 2}$ only in first and last states and no state depends on both $\theta_{i, 4}^{l}$ and $\theta_{i, 5}^{l}$.

\section{CONCLUSION}

This work provided methods for tuning the parameters of T1 FLS and an IT2 FLS, which made T1 and T2 FLSs much more accessible to FLS modelers by using mathematical formulas. We have modeled T1 FLSs when a collection of training data is available. Further, presented an application to derive the SDTFM depending on generalized bell-shaped MF for the antecedent and the consequent. Depending on general formula of SDTFM, we have proposed an IT2 NS-T2 FLS in order to determine all the parameters of the antecedent and consequent MFs using the set of $n$ input-output and we have built mathematical formulas to calculate the derivatives $\partial \cosh (\alpha) / \partial \theta$. Present general formulas for the left and right end-points of the type-reduced set. Proposed mathematical formulas for derivatives of $\partial \cosh (\alpha)$ with respect to antecedent MF parameters and consequent MF parameters. As well as, provided an algorithm of the derivatives for antecedent parameters to calculate $\partial \cosh (\alpha) / \partial \theta_{i, k}^{l}$. An application is provided and showed how to complete the calculations for input measurement and antecedent Gaussian primary MFs with uncertain standard deviations and means. From the result of the application, note that $\underline{\mu}_{\hat{\sigma}_{i}^{l}}\left(\underline{x}_{i, S}^{l}\right)$ depends on $\theta_{i, 4}^{l}$ in all states, and $\bar{\mu}_{\hat{o}_{i}^{l}}\left(\bar{x}_{i, s}^{l}\right)$ depends on $\theta_{i, 5}^{l}$ only in first and last states and no state depends on both $\theta_{i, 4}^{l}$ and $\theta_{i, 5}^{l}$. Future studies will try to handle, the large number of parameters or reducing the number of model parameters. If some parameters are shared across rules or MFs and if mathematical formulas for derivatives cannot be obtained then we must be modify and control some or all of the results of this work.

\section{APPENDIX A}

\section{Re-expressing $y_{l}$ in Rule-Ordered Format}

In order to re-express $y_{l}$ in terms of rule-ordered quantities, we have to re-express the four sums of $y_{l}$ in (6) as the follows [14]:

Let $\underline{\mathbf{a}}=\left(\underline{a}^{1}, \underline{a}^{2}, \ldots, \underline{a}^{M}\right)^{T}, \overline{\mathbf{a}}=\left(\bar{a}^{1}, \bar{a}^{2}, \ldots, \bar{a}^{M}\right)^{T}$,

$\underline{\mathbf{b}}=\left(\underline{b}^{1}, \underline{b}^{2}, \ldots, \underline{b}^{M}\right)^{T} \equiv Q_{l}$. $\underline{\mathbf{a}}$ and $\overline{\mathbf{b}}=\left(\bar{b}^{1}, \bar{b}^{2}, \ldots, \bar{b}^{M}\right)^{T} \equiv Q_{l} \cdot \overline{\mathbf{a}}$,

$\mathbf{E}_{\mathbf{1}}=\left(e_{1}\left|e_{2}\right| \ldots\left|e_{L}\right| 0|\ldots| 0\right)_{L \times M}^{T}$, where $e_{i}=L \times 1$ is the $i^{t h}$ elementary vector, and

$\mathbf{E}_{2}=\left(0|\ldots| 0\left|\varepsilon_{1}\right| \varepsilon_{2}|\ldots| \varepsilon_{M-L}\right)_{(M-L) \times 1}^{T}$ where $\varepsilon_{i}=(M-L) \times$ 1 elementary vector, therefore

$$
\begin{aligned}
\sum_{i=1}^{L} \bar{b}^{i} y_{l}^{i}= & \left(e_{1}\left|e_{2}\right| \ldots\left|e_{L}\right| 0|\ldots| 0\right)^{T}\left(\bar{b}^{1}, \bar{b}^{2}, \ldots, \bar{b}^{M}\right)^{T} * \\
& \left(e_{1}\left|e_{2}\right| \ldots\left|e_{L}\right| 0|\ldots| 0\right)\left(y_{l}^{1}, \ldots, y_{l}^{M}\right) \\
= & \left(\mathbf{E}_{\mathbf{1}} \overline{\mathbf{b}}\right)^{T}\left(\mathbf{E}_{\mathbf{1}} y_{l}\right)=\left(\mathbf{E}_{\mathbf{1}} Q_{l} \cdot \overline{\mathbf{a}}\right)^{T}\left(\mathbf{E}_{\mathbf{1}} Q_{l} \cdot z_{l}\right) \\
= & \overline{\mathbf{a}}^{T} \underbrace{Q_{l}{ }^{T} \mathbf{E}_{\mathbf{1}}{ }^{T} \mathbf{E}_{\mathbf{1}} Q_{l}} z_{l}=\overline{\mathbf{a}}^{T} \underbrace{H_{l 1} z_{l}}=\overline{\mathbf{a}}^{T} \sigma_{l} .
\end{aligned}
$$

$\sum_{i=L+1}^{M} y_{l}^{i} \underline{b}^{i}=\left(0|\ldots| 0\left|\varepsilon_{1}\right| \varepsilon_{2}|\ldots| \varepsilon_{M-L}\right)^{T}\left(\underline{b}^{1}, \underline{b}^{2}, \ldots, \underline{b}^{M}\right)^{T} *$

$$
\begin{aligned}
& \left(0|\ldots| 0\left|\varepsilon_{1}\right| \varepsilon_{2}|\ldots| \varepsilon_{M-L}\right)\left(y_{l}^{1}, \ldots, y_{l}^{M}\right) \\
= & \left(\mathbf{E}_{2} \underline{\mathbf{b}}\right)^{T}\left(\mathbf{E}_{2} y_{l}\right)=\left(\mathbf{E}_{2} Q_{l} \cdot \underline{\mathbf{a}}\right)^{T}\left(\mathbf{E}_{2} Q_{l} \cdot z_{l}\right) \\
= & \underline{\mathbf{a}}^{T} \underbrace{Q_{l}{ }^{T} \mathbf{E}_{2}{ }^{T} \mathbf{E}_{2} Q_{l}} z_{l}=\underline{\mathbf{a}}^{T} \underbrace{H_{l 2} z_{l}}=\underline{\mathbf{a}}^{T} \rho_{l} .
\end{aligned}
$$

in which $\left(Q_{l}{ }^{T} \mathbf{E}_{\mathbf{1}}{ }^{T} \mathbf{E}_{\mathbf{1}} Q_{l}\right)_{M \times M}=H_{l 1},\left(H_{l 1} z_{l}\right)_{M \times 1}=\sigma_{l}$,

$\left(Q_{l}{ }^{T} \mathbf{E}_{2}{ }^{T} \mathbf{E}_{2} Q_{l}\right)_{M \times M}=H_{l 2}$, and $\left(H_{l 2} z_{l}\right)_{M \times 1}=\rho_{l}$

Suppose $(\underbrace{1,1, \ldots, 1}_{L}, 0, \ldots, 0)_{M \times 1}=k_{1 l}$ and $(0, \ldots, 0, \underbrace{1,1, \ldots, 1}_{M-L})_{M \times 1}=$

$k_{2 l} \sum_{i=1}^{L} \bar{b}^{i}=(1,1, \ldots, 1,0, \ldots, 0)^{T}\left(\bar{b}^{1}, \bar{b}^{2}, \ldots, \bar{b}^{M}\right)$

$$
=\underbrace{k_{1 l}^{T} Q_{l}} \cdot \overline{\mathbf{a}}=\tau_{l}^{T} \overline{\mathbf{a}}
$$

$\sum_{i=L+1}^{M} \underline{b}^{i}=(0, \ldots, 0,1,1, \ldots, 1)^{T}\left(\underline{b}^{1}, \underline{b}^{2}, \ldots, \underline{b}^{M}\right)$

$$
=\underbrace{k_{2 l}{ }^{T} Q_{l}} \cdot \underline{\mathbf{a}}=\vartheta_{l}^{T} \underline{\mathbf{a}}
$$

in which $\left(k_{1 l}{ }^{T} Q_{l}\right)_{1 \times M}=\tau_{l}$ and $\left(k_{2 l}{ }^{T} Q_{l}\right)_{1 \times M}=\vartheta_{l}$.

Consequently, from (A-1)-(A-4) we are obtained

$$
\begin{aligned}
y_{l} & =\frac{\overline{\mathbf{a}}^{T} \boldsymbol{\sigma}_{l}+\underline{\mathbf{a}}^{T} \boldsymbol{\rho}_{l}}{\boldsymbol{\tau}_{l}^{T} \overline{\mathbf{a}}+\boldsymbol{\vartheta}_{l}^{T} \underline{\mathbf{a}}}=\frac{\overline{\mathbf{a}}^{T} \boldsymbol{\sigma}_{l}+\underline{\mathbf{a}}^{T} \boldsymbol{\rho}_{l}}{\overline{\mathbf{a}}^{T} \boldsymbol{\tau}_{l}+\underline{\mathbf{a}}^{T} \boldsymbol{\vartheta}_{l}} \\
& =\frac{\sum_{i=1}^{M} \sigma_{l, i} \bar{b}^{i}+\sum_{i=1}^{M} \rho_{l, i} \underline{b}^{i}}{\sum_{i=1}^{M} \tau_{l, i} \bar{b}^{i}+\sum_{i=1}^{M} \vartheta_{l, i} \underline{b}^{i}}
\end{aligned}
$$


Note that Equation (A-5) includes the entire $\overline{\mathbf{a}}, \underline{\mathbf{a}}$ and $z_{l}$ vectors. The matrices $H_{l 1}, H_{l 2}$ and the vectors $k_{1 l}, k_{2 l}$ will automatically get out of the unnecessary elements of $\overline{\mathbf{a}}$ and $\underline{\mathbf{a}}$ those depend on $L$.

\section{ACKNOWLEDGMENTS}

The author wishes to thank the reviewers for their excellent suggestions that have been incorporated into this paper. I would like to acknowledge my profound gratitude to my research supervisors DR. Sanjeev Kumar, and DR. M. S. Saroa for their vigilant supervision as well as for pouring many invaluable pearls from the deep ocean of knowledge.

Table I. Definitions of the states and the $\underline{\mu}_{\widehat{o}_{i}^{l}}\left(\underline{x}_{i, s}^{l}\right)$ and $\bar{\mu}_{\widehat{o}_{i}^{l}}\left(\bar{x}_{i, s}^{l}\right)$

\begin{tabular}{|c|c|c|}
\hline State of $\boldsymbol{x}_{\boldsymbol{i}}^{\prime}$ & $\underline{\boldsymbol{\mu}}_{\widehat{\boldsymbol{\sigma}}_{\boldsymbol{i}}^{l}\left(\underline{\boldsymbol{x}}_{\boldsymbol{i}, \boldsymbol{S}}^{l}\right)}$ & $\overline{\boldsymbol{\mu}}_{\widehat{\boldsymbol{o}}_{\boldsymbol{i}}^{l}}\left(\overline{\boldsymbol{x}}_{\boldsymbol{i}, \boldsymbol{s}}^{l}\right)$ \\
\hline$x_{i}^{(j)}<\left(\frac{m_{i 1}^{l}+m_{i 2}^{l}}{2}-\frac{\sigma_{i 1}\left(m_{i 2}^{l}-m_{i 1}^{l}\right)}{2 \sigma_{i}^{l}}\right) \leq m_{i 1}^{l}$ & $\exp \left(-\frac{\left(m_{i 2}^{l}-x_{i}^{(j)}\right)^{2}}{2\left(\sigma_{i 1}+\sigma_{i}^{l}\right)}\right)$ & $\exp \left(-\frac{\left(m_{i 1}^{l}-x_{i}^{(j)}\right)^{2}}{2\left(\sigma_{i 2}+\sigma_{i}^{l}\right)}\right)$ \\
\hline$m_{i 1}^{l} \leq x_{i}^{(j)}<\left(\frac{m_{i 1}^{l}+m_{i 2}^{l}}{2}-\frac{\sigma_{i 1}\left(m_{i 2}^{l}-m_{i 1}^{l}\right)}{2 \sigma_{i}^{l}}\right) \leq m_{i 2}^{l}$ & $\exp \left(-\frac{\left(m_{i 2}^{l}-x_{i}^{(j)}\right)^{2}}{2\left(\sigma_{i 1}+\sigma_{i}^{l}\right)}\right)$ & 1 \\
\hline$m_{i 1}^{l} \leq\left(\frac{m_{i 1}^{l}+m_{i 2}^{l}}{2}-\frac{\sigma_{i 1}\left(m_{i 2}^{l}-m_{i 1}^{l}\right)}{2 \sigma_{i}^{l}}\right) \leq x_{i}^{(j)} \leq\left(\frac{m_{i 1}^{l}+m_{i 2}^{l}}{2}+\frac{\sigma_{i 1}\left(m_{i 2}^{l}-m_{i 1}^{l}\right)}{2 \sigma_{i}^{l}}\right) \leq m_{i 2}^{l}$ & $\exp \left(-\frac{\left(m_{i 2}^{l}+m_{i 1}^{l}-2 x_{i}^{(j)}\right)^{2}}{8 \sigma_{i 1}}-\frac{\left(m_{i 2}^{l}-m_{i 1}^{l}\right)^{2}}{8 \sigma_{i}^{l}}\right)$ & 1 \\
\hline$m_{i 1}^{l} \leq\left(\frac{m_{11}^{l}+m_{i 2}^{l}}{2}+\frac{\sigma_{i 1}\left(m_{21}^{l}-m_{i 1}^{l}\right)}{2 \sigma_{i}^{l}}\right)<x_{i}^{(j)} \leq m_{i 2}^{l}$ & $\exp \left(-\frac{\left(m_{i 1}^{l}-x_{i}^{(j)}\right)^{2}}{2\left(\sigma_{i 1}+\sigma_{i}^{l}\right)}\right)$ & 1 \\
\hline$\left(\frac{m_{i 1}^{l}+m_{i 2}^{l}}{2}+\frac{\sigma_{i 1}\left(m_{i 2}^{l}-m_{i 1}^{l}\right)}{2 \sigma_{i}^{l}}\right)<m_{i 2}^{l} \leq x_{i}^{(j)}$ & $\exp \left(-\frac{\left(m_{i 1}^{l}-x_{i}^{(j)}\right)^{2}}{2\left(\sigma_{i 1}+\sigma_{i}^{l}\right)}\right)$ & $\exp \left(-\frac{\left(m_{i 2}^{l}-x_{i}^{(j)}\right)^{2}}{2\left(\sigma_{i 2}+\sigma_{i}^{l}\right)}\right)$ \\
\hline
\end{tabular}


Table II. The derivatives of $\underline{\mu}_{\widehat{\sigma}_{i}^{l}}\left(\underline{x}_{i, s}^{l}\right)$ with respect to all $\theta_{i, j}^{l}$

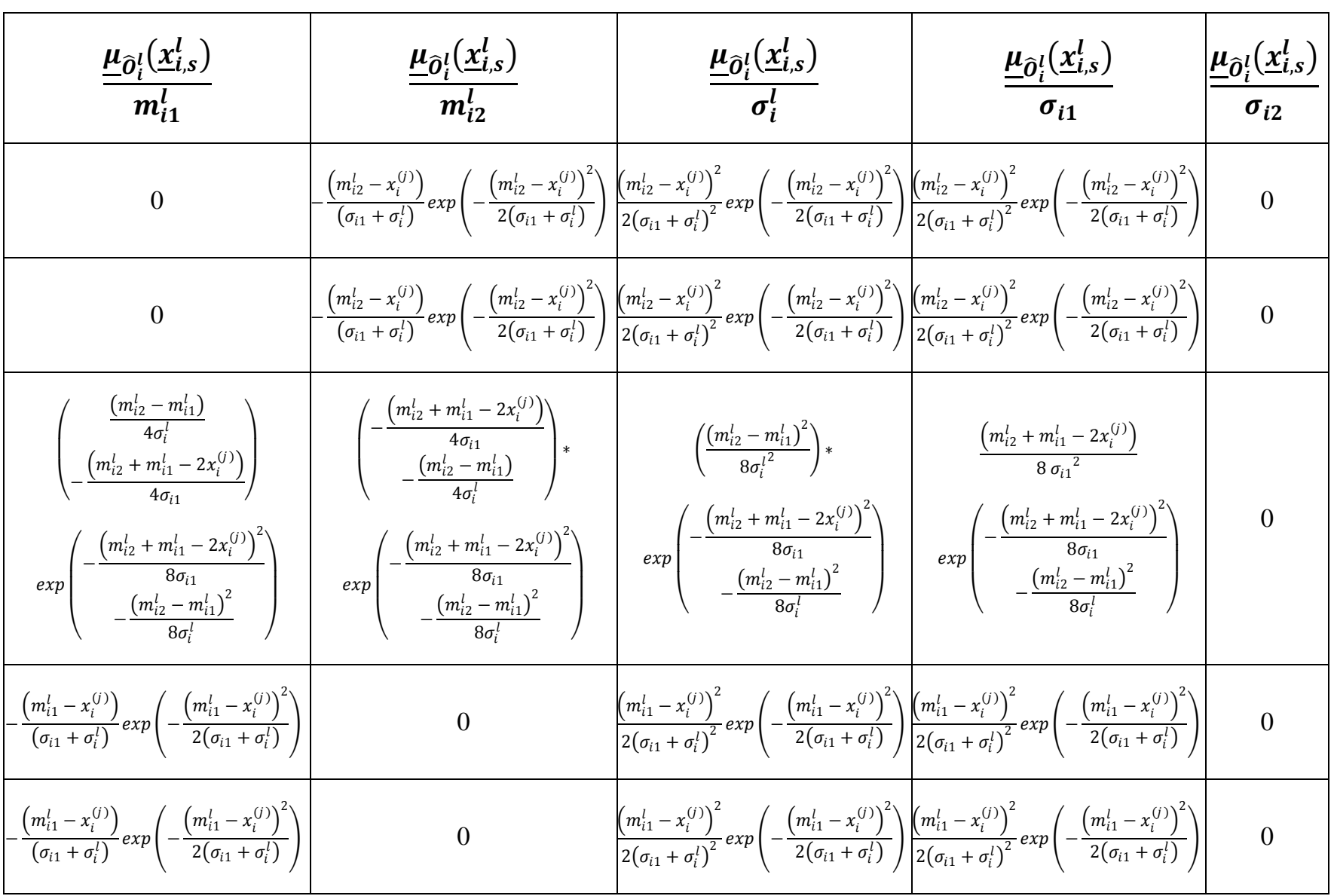

Table III. The derivatives of $\bar{\mu}_{\widehat{o}_{i}^{l}}\left(\bar{x}_{i, s}^{l}\right)$ with respect to all $\theta_{i, j}^{l}$

\begin{tabular}{|c|c|c|c|c|}
\hline$\frac{\bar{\mu}_{\widehat{o}_{i}^{l}}\left(\bar{x}_{i, s}^{l}\right)}{m_{i 1}^{l}}$ & $\frac{\bar{\mu}_{\widehat{o}_{i}^{l}}\left(\bar{x}_{i, s}^{l}\right)}{m_{i 2}^{l}}$ & $\frac{\overline{\boldsymbol{\mu}}_{\widehat{\boldsymbol{o}}_{i}^{l}}\left(\overline{\boldsymbol{x}}_{i, s}^{l}\right)}{\sigma_{i}^{l}}$ & $\frac{\overline{\boldsymbol{\mu}}_{\widehat{\boldsymbol{o}}_{i}^{l}}\left(\overline{\boldsymbol{x}}_{\boldsymbol{i}, \mathrm{s}}^{l}\right)}{\sigma_{i 1}}$ & $\frac{\bar{\mu}_{\widehat{o}_{i}^{l}}\left(\bar{x}_{i, s}^{l}\right)}{\sigma_{i 2}}$ \\
\hline$-\frac{\left(m_{i 1}^{l}-x_{i}^{(j)}\right)}{\left(\sigma_{i 2}+\sigma_{i}^{l}\right)} \exp \left(-\frac{\left(m_{i 1}^{l}-x_{i}^{(j)}\right)^{2}}{2\left(\sigma_{i 2}+\sigma_{i}^{l}\right)}\right)$ & 0 & $\frac{\left(m_{i 1}^{l}-x_{i}^{(j)}\right)}{2\left(\sigma_{i 2}+\sigma_{i}^{l}\right)^{2}} \exp \left(-\frac{\left(m_{i 1}^{l}-x_{i}^{(j)}\right)^{2}}{2\left(\sigma_{i 2}+\sigma_{i}^{l}\right)}\right)$ & 0 & $\frac{\left(m_{i 1}^{l}-x_{i}^{(j)}\right)}{2\left(\sigma_{i 2}+\sigma_{i}^{l}\right)^{2}} \exp \left(-\frac{\left(m_{i 1}^{l}-x_{i}^{(j)}\right)^{2}}{2\left(\sigma_{i 2}+\sigma_{i}^{l}\right)}\right)$ \\
\hline 0 & 0 & 0 & 0 & 0 \\
\hline 0 & 0 & 0 & 0 & 0 \\
\hline 0 & 0 & 0 & 0 & 0 \\
\hline 0 & $\frac{\left(m_{i 2}^{l}-x_{i}^{(j)}\right)}{\left(\sigma_{i 2}+\sigma_{i}^{l}\right)} \exp \left(-\frac{\left(m_{i 2}^{l}-x_{i}^{(j)}\right)^{2}}{2\left(\sigma_{i 2}+\sigma_{i}^{l}\right)}\right.$ & $\frac{\left(m_{i 2}^{l}-x_{i}^{(j)}\right)^{2}}{2\left(\sigma_{i 2}+\sigma_{i}^{l}\right)^{2}} \exp \left(-\frac{\left(m_{i 2}^{l}-x_{i}^{(j)}\right)^{2}}{2\left(\sigma_{i 2}+\sigma_{i}^{l}\right)}\right.$ & 0 & $\frac{\left(m_{i 2}^{l}-x_{i}^{(j)}\right)^{2}}{2\left(\sigma_{i 2}+\sigma_{i}^{l}\right)^{2}} \exp \left(-\frac{\left(m_{i 2}^{l}-x_{i}^{(j)}\right)^{2}}{2\left(\sigma_{i 2}+\sigma_{i}^{l}\right)}\right.$ \\
\hline
\end{tabular}




\section{REFERENCE}

[1] Agüero J., Vargas A., 2007, "Calculating functions of interval type-2 fuzzy numbers for fault current analysis", IEEE Transactions on Fuzzy Systems, vol. 15, no. 1, pp. 3140.

[2] Coupland S., John R., 2007, "Geometric type-1 and type-2 fuzzy logic systems," IEEE Transactions on Fuzzy Systems, vol. 15 , no. 1 , pp. 3-15

[3] Castillo, O., Melin P., 2012, "Recent Advances in Interval Type-2 Fuzzy Systems," Springer Briefs in Applied Sciences and Technology

[4] Hndoosh, R., Saroa, M., and Kumar, S., 2012, "Fuzzy and adaptive neuro-fuzzy inference system of washing machine", European Journal of Scientific Research, vol. 86, no. $3,443-459$.

[5] Hndoosh, R., Saroa, M., and Kumar, S., 2013, "A propo sition for using mathematical models based on a fuzzy system with application", International Journal of Mathematical Sciences, vol. 33, no. 2, 1356-1374.

[6] Hndoosh, R., Saroa, M., and Kumar, S., 2014, "Mathe matical structure of fuzzy modeling of medical diagnoses by using clustering models", International Journal of Scientific \& Engineering Research, vol. 5, no. 8, 545-554.

[7] Hndoosh, R., Saroa, M., and Kumar, S., 2014, "Fuzzy mathematical model for detection of lung cancer using a multi-NFCLass with confusion fuzzy matrix for accuracy, International Journal of Mathematical and Computer Modeling, vol. 19, no. 1, 1129-1141.

[8] Hndoosh, R., Saroa, M., and Kumar, S., 2014, "Fuzzy mathematical models for the analysis of fuzzy systems with application to liver disorders, IOSR Journal of Computer Engineering, vol. 10 no. 5, pp. 1129-1141.

[9] Juang C., Tsao Y., 2008, "A self-evolving interval type-2 fuzzy neural network with online structure and parameter learning," IEEE Transactions on Fuzzy Systems, vol. 16, no. 6, PP. 1411-1424.

[10] Karnik N., Mendel J., Liang Q., 1999, “Type-2 fuzzy logic systems," IEEE Transactions on Fuzzy Systems, vol. 7, no. 6, PP. 643-658.

[11] Karnik N., Mendel J., 1998, "Introduction to type-2 fuzzy logic systems," IEEE International Conference on Fuzzy Systems Proceedings, pp. 915-920.

[12] Liang, Q., Mendel J., 2000, "Interval type-2 fuzzy logic systems: theory and design," IEEE Transactions on Fuzzy Systems, vol. 8, no. 5, pp. 535-550.

[13] Mendel J., 2004, "Computing derivatives in interval type-2 fuzzy logic systems," IEEE Transactions on Fuzzy Systems, vol. 12 , no. 1 , pp. 84-98.

[14] Mendel J., 2009, "On answering the question "Where do i start in order to solve a new problem involving interval type-2 fuzzy sets?", International Journal of Information Sciences, vol. 179, pp. 3418-3431
[15] Mendel J., 2007, "Type-2 fuzzy sets and systems: an overview," IEEE computation intelligence magazine, vol. 2, no. 1 , pp. 20-29.

[16] Mendel J., 2007, "Advances in type-2 fuzzy sets and systems," International Journal of Information Sciences, vol. 177 , pp. 84-110.

[17] Mendel J., John R., Liu F., 2006, "Interval type-2 fuzzy logic systems made simple," IEEE Transactions on Fuzzy Systems, vol. 14 , no. 6, pp. 808-821.

[18] Mendel J., 2004, "Computing derivatives in interval type-2 fuzzy logic systems," IEEE Transactions on Fuzzy Systems, vol. 12, no. 1, pp. 84-98.

[19] Mendel J., 2001, "Uncertain Rule-Based Fuzzy Logic Systems: Introduction and New Directions", Prentice Hall PTR, Upper Saddle River NJ.

[20] Miguel P., Carlos L., Javier F., Edurne B., Humberto B., 2013, "Interval type-2 fuzzy sets constructed from several membership functions: application to the fuzzy thresholding algorithm," IEEE Transactions on Fuzzy Systems, vol. 21, no. 2, PP. 230-244.

[21] Mitchell H., 2006, "Ranking type-2 fuzzy numbers," IEEE Transactions on Fuzzy Systems, vol. 14, no. 2, pp. 287-294.

[22] Molaeezadeh S., Moradi M., 2013, “A function representation for non-uniform type-2 fuzzy sets: theory and design," International Journal of Approximate Reasoning, vol. 54, pp. 273-289.

[23] Ondrej, L., Milos M., 2010, "Importance sampling based defuzzification for general type-2 fuzzy sets," IEEE World Congress on Computational Intelligence, vol. 10, pp. 19431949.

[24] Starczewski, J., 2009, "Efficient triangular type-2 fuzzy logic systems," International Journal of Approximate Reasoning, vol. 50, pp. 799-811.

[25] Wagner, C., Hagras, H. 2010, “Toward general type-2 fuzzy logic systems based on zslices," IEEE Transactions on Fuzzy Systems, vol. 18, no. 4, PP. 637-660.

[26] Wu, D., Tan, W., 2006, "Genetic learning and performance evaluation of interval type-2 fuzzy logic controllers," Engineering Applications of Artificial Intelligence, vol. 19, pp. 829-841.

[27] Wu, H., Mendel J., 2002, "Uncertainty bounds and their use in the design of interval type-2 fuzzy logic systems," IEEE Transactions on Fuzzy Systems, vol. 10, no. 5, pp. 622-639.

[28] Yeh C., Jeng W., Lee S., 2011, "An enhanced typereduction algorithm for type-2 fuzzy sets," IEEE Transactions on Fuzzy Systems, vol. 19, no. 2, PP. 227-240.

[29] Zakaria R., 2013, "On defining complex uncertainty data points by type-2 fuzzy number: two specials cases," Int Journal of Math Analysis, vol. 7, no. 26, PP. 1285 - 1300.

[30] Zenga J., Xieb L., Liu Z., 2008, “Type-2 fuzzy Gaussian mixture models," The Journal of the Pattern Recognition Society, vol. 41, pp. $3636-3643$. 